OUNP-99-04

MZ-TH-99-02

\title{
High- $Q^{2}$ Deep Inelastic Scattering at HERA
}

\author{
A M Cooper-Sarkar \\ Department of Physics, Univeristy of Oxford, Oxford OX1 3RH, UK
}

\begin{abstract}
A Bodek
Department of Physics and Astronomy, University of Rochester, Rochester, NY 14627
\end{abstract}

K Long

Imperial College of Science and Technology, London SW7 2BZ,UK

E Rizvi

School of Physics, Birmingham University, Birmingham B15 2TT, UK

\section{H Spiesberger}

Institut für Physik, Johannes-Gutenberg-Universität D-55099 Mainz, Germany

\begin{abstract}
High $Q^{2} \mathrm{NC}$ and $\mathrm{CC}$ cross sections as measured at HERA can give information on two distinct areas of current interest. Firstly, supposing that all the electroweak parameters are well known, these cross-sections may be used to give information on parton distribution functions (PDFs) in the new kinematic regime at high $x$ and high $Q^{2}$. Secondly, supposing that PDFs are well known after evolution in $Q^{2}$ from the kinematic regime where they are already measured, these cross-sections may be used to give information on electroweak parameters in a process where the exchanged boson is 'spacelike' rather than 'timelike'. WG1 addressed itself to clarifying the limits of our present and possible future knowledge on both these points.
\end{abstract}

\section{Introduction}

The differential (Born) cross-section for charged lepton-nucleon scattering, mediated by the neutral current at high $Q^{2}$ and accounting for the possible polarization of the lepton beam, is given by

$$
\frac{d^{2} \sigma\left(l^{ \pm} N\right)}{d x d Q^{2}}=\frac{2 \pi \alpha^{2}}{Q^{4} x}\left[Y_{+} F_{2}^{l N}\left(x, Q^{2}\right) \mp Y_{-} x F_{3}^{l N}\left(x, Q^{2}\right)\right],
$$

where $F_{2}$ is expressed in terms of parton distributions as

$$
F_{2}^{l N}\left(x, Q^{2}\right)=\Sigma_{i} A_{i}^{L, R}\left(Q^{2}\right) \times\left(x q_{i}\left(x, Q^{2}\right)+x \bar{q}_{i}\left(x, Q^{2}\right)\right)
$$


and the sum is taken over flavours of quarks which are above threshold. (A contribution from the longitudinal structure function, $F_{L}$, is neglected in Eq. 1). $A_{i}$ gives the couplings of the quarks and leptons to the currents

$$
A_{i}^{L, R}\left(Q^{2}\right)=e_{i}^{2}+2 e_{i} e_{l}\left(v_{l} \pm a_{l}\right) v_{i} P_{Z}+\left(v_{l} \pm a_{l}\right)^{2}\left(v_{i}^{2}+a_{i}^{2}\right) P_{Z}^{2}
$$

These couplings depend on whether the polarization of the lepton beam is left $(L$, upper signs) or right $\left(R\right.$, lower signs) handed. The notation $e_{l}$ specifies the incoming lepton's charge such that $e_{l}=-1$. The vector and axial-vector couplings of the fermions are given by

$$
v_{f}=\left(T_{3 f}-2 e_{f} \sin ^{2} \theta_{W}\right), \quad a_{f}=T_{3 f}
$$

where the definition holds good for any fermion, whether lepton or quark; $T_{3 f}$ is the weak

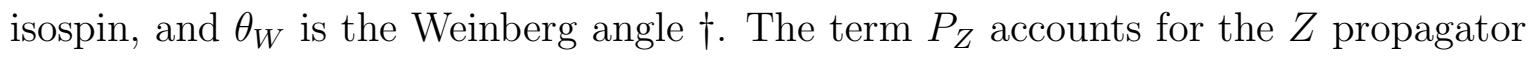

$$
P_{Z}=\frac{Q^{2}}{Q^{2}+M_{Z}^{2}} \frac{\sqrt{2} G_{\mu} M_{Z}^{2}}{4 \pi \alpha}
$$

Thus one can identify the contributions of $\gamma$ exhange, $\gamma-Z$ interference and $Z$ exchange in the coupling $A_{i}$. Finally the parity violating structure function $x F_{3}$ is given by

$$
x F_{3}^{l N}\left(x, Q^{2}\right)=\Sigma_{i} B_{i}^{L, R}\left(Q^{2}\right) \times\left(x q_{i}\left(x, Q^{2}\right)-x \bar{q}_{i}\left(x, Q^{2}\right)\right),
$$

where

$$
B_{i}^{L, R}\left(Q^{2}\right)=\mp 2 e_{i} e_{l}\left(v_{l} \pm a_{l}\right) a_{i} P_{Z} \pm 2\left(v_{l} \pm a_{l}\right)^{2} v_{i} a_{i} P_{Z}^{2}
$$

The corresponding cross-sections for antilepton scattering are given by swapping $L \rightarrow R$, $R \rightarrow L$ in the expressions for $F_{2}$ and $x F_{3}$ given in Eq. 2 and Eq. 6, and by substituting the antilepton charge $e_{l}=+1$.

The differential (Born) cross-sections for charged lepton-nucleon scattering, mediated by the charged current are given by

$$
\frac{d^{2} \sigma^{C C}\left(l^{ \pm} N\right)}{d x d Q^{2}}=\frac{G_{\mu}^{2}}{4 \pi x} \frac{M_{W}^{4}}{\left(Q^{2}+M_{W}^{2}\right)^{2}}\left[Y_{+} F_{2}\left(x, Q^{2}\right) \mp Y_{-} x F_{3}\left(x, Q^{2}\right)\right] .
$$

Expressing the structure functions in terms of parton distributions and accounting for polarization of the lepton beam gives

$\frac{d^{2} \sigma^{C C}\left(l^{-} N\right)}{d x d Q^{2}}=(1-P) \frac{G_{\mu}^{2}}{2 \pi x} \frac{M_{W}^{4}}{\left(Q^{2}+M_{W}^{2}\right)^{2}}\left[\Sigma_{i=u, c} x q_{i}\left(x, Q^{2}\right)+(1-y)^{2} \Sigma_{i=d, s} x \bar{q}_{i}\left(x, Q^{2}\right)\right](9)$

whereas for antilepton scattering we have

$\left.\frac{d^{2} \sigma^{C C}\left(l^{+} N\right)}{d x d Q^{2}}=(1+P) \frac{G_{\mu}^{2}}{2 \pi x} \frac{M_{W}^{4}}{\left(Q^{2}+M_{W}^{2}\right)^{2}}\left[(1-y)^{2} \Sigma_{i=d, s} x q_{i}\left(x, Q^{2}\right)+\Sigma_{i=u, c} x \bar{q}_{i}\left(x, Q^{2}\right)\right] 10\right)$

where the sums contain only the appropriate quarks or antiquarks for the charge of the current and the polarization of the lepton beam, $P=\left(N_{R}-N_{L}\right) /\left(N_{R}+N_{L}\right)$.

$\dagger$ Neutrinos and charged leptons of the same family form weak isospin doublets with $T_{3}=1 / 2,-1 / 2$ respectively; and the quarks form similar weak isospin doublets, within the families $(u, d),(c, s),(t, b)$, with $T_{3}=1 / 2,-1 / 2$ respectively. For antiparticles both $T_{3 f}$ and $e_{f}$ change sign. 
Clearly measurements of these cross-sections can give information both on electroweak parameters and on parton distribution functions (PDFs). In Sec.2 and Sec.3 we consider how accurately we really know PDFs based on lower $Q^{2}$ measurements, and hence how much HERA high $Q^{2}$ measurements will improve our knowledge. Searches for new physics rely crucially on accurate determinations of event rates or cross-sections from standard model (SM) processes. In lepton-hadron or hadron-hadron collisions, this translates into a need to estimate uncertainties on the PDFs of the incident hadron. An example of such a need was seen when in 1997 both HERA experiments observed a slight excess of events at very high $Q^{2}[1]$. Uncertainties on the SM cross-sections have been obtained by varying the input PDFs in the Monte-Carlo models. This method may not reflect the true PDF uncertainty, but rather highlight the different approaches used by global fitters in their PDF extractions. More reliable estimates of the uncertainties on PDFs are considered in Sec. 2.

Recent work on parton distributions functions (PDF's) in the nucleon has focussed on probing the sea and gluon distribution at small $x$. The valence quark distributions have been considered to be relatively well understood. However, this is not really true at $x \gtrsim 0.5$. In particular our knowledge of the $d$ quark distribution at high $x$ is extracted from data taken on deuteron targets, and nuclear binding effects in the deuteron have not always been properly accounted. Our knowledge of the $u$ quark density at high $x$ has also been questioned by Kuhlmann et al, who recently proposed a toy model [2] which included the possibility of an additional contribution to the $u$ quark distribution (beyond $x>0.75$ ) as an explanation for both the initial HERA high $Q^{2}$ anomaly [ [ $]$ ] and for the jet excess at high- $P_{T}$ at CDF [3]. A precise knowledge of the $u$ and $d$ quark distribution at high $x$ is very important at collider energies in order to estimate backgrounds to signals for new physics accurately. In addition, the value of $d / u$ as $x \rightarrow 1$ is of theoretical interest in its own right. It is clear from Eqs. 9 and 10 that HERA CC data can greatly improve our knowledge of these high $x$ valence quark densities. We discuss the current status of our knowledge in Sec. 3 .

In Sec. 1 and Sec. 5 we discuss the possibilty of using high $Q^{2}$ HERA data to gain information on electroweak parameters. We have specified the Born cross-sections for the processes above but if we aim for an eventual precision of $1 \%$ then electroweak radiative corrections of order $O(\alpha)$ have to be taken into account. We discuss the current status of implementation of the full electroweak radiative corrections and the implications for fitting electroweak parameters in Sec. 4 .

Measurements from LEP and the Tevatron tightly constrain the electroweak sector of the Standard Model (SM). In particular, the mass of the $W$-boson, $M_{W}$, is known precisely from measurements of the properties of real, 'time-like', $W$-bosons. In the SM description of charged current (CC) deep inelastic scattering (DIS) this same mass appears in the 'propagator' of the exchanged 'space-like' $W$-boson (see Eq. \&). Hence, for the SM to be self-consistent the same value of $M_{W}$ must be obtained from measurements of the space-like 'propagator mass' in CC DIS as is obtained from LEP and the Tevatron; any difference indicates new physics. In Sec. 5 we discuss the present and future 
achievable accuracy of fits to $M_{W}$ and $G_{\mu}$ using HERA data.

The workshop necessarily concerned itself with results which can be achieved at present or in the near future. In Sec. 6] we give a summary and discuss prospects for the more distant future.

\section{Uncertainties on Parton Distributions}

\subsection{Experimental Uncertainties}

Measurements of cross-sections and structure functions inevitably involve detailed estimates of systematic uncertainties which are usually published with the measurements. The systematic uncertainties may be classified into two types: uncorrelated systematics in which the uncertainties fluctuate point-to-point (for example limitations on the statistics generated in Monte Carlo simulations of systematic effects) and correlated systematics which affect the data points in a coherent manner (for example, uncertainty in the luminosity determination). These systematic uncertainties may be propagated into the QCD fits used to extract parton densities and thereby allow an estimate to be made of experimental uncertainties in the extracted PDFs. A detailed derivation of the formalism necessary to make such estimates is given in [4] and both the $\mathrm{H} 1$ and ZEUS collaborations have propagated systematic errors into their estimates of the errors on the gluon density at low $x$ [5, 6]. These experimental uncertainties are not the focus of the present section. They are thoroughly discussed by Botje [7].

\section{2. 'Theoretical' Uncertainties}

In addition to experimental uncertainties there are uncertainties which are of a more theoretical nature. A list of such sources of uncertainty is given below.

- $\boldsymbol{\alpha}_{\boldsymbol{s}}$. The size of $\alpha_{s}$ governs the NLO $Q^{2}$ evolution of the PDFs. The Particle Data Group estimates an uncertainty of \pm 0.002 at a scale of $M_{Z}^{2}$ [8].

- DGLAP solution methods. The DGLAP equations may be solved numerically using two classes of methods, namely $x$-space methods and $n$-space methods. Differences between these two approaches are generally small, but can be as large as $7 \%$ for the gluon density at low $x$ [9]

- Heavy Quark Treatment. The way that heavy quarks are treated in DGLAP evolution can lead to uncertainties. In one approach the heavy quarks $(c, b)$ are treated as massless partons and are evolved only above $Q^{2}$ thresholds which are usually set equal to the masses squared. This method is not expected to give an accurate description of the data in the region of low $Q^{2}$, where threshold effects appear as $\ln Q^{2} / M_{c}^{2}$. In this region heavy quarks are considered as generated by the boson-gluon fusion process and the method of [10] is used. This method treats the mass logarithms correctly, but fails to match the asymptotic limit when $Q^{2} \gg M_{c}^{2}$. The recent work of Lai et. al [11], Martin et. al [12] and Buza et. al. [13] aims 
to have a smooth transition from the threshold region to the asymptotic limit, but such an approach has not yet been implemented in the fitting programs used by the experimental collaborations.

- Target mass corrections. The identification of $x$ with the fraction of the nucleon's momentum taken by the struck quark cannot be maintained when $Q^{2} \simeq M^{2}$ (where $M$ is the nucleon mass) and corrections to the formulae of the form $x^{2} \cdot M^{2} / Q^{2}$ are necessary [14].

- Higher Twist effects. More complicated interactions involving more than one parton, like secondary interactions of quarks with the proton remnant, give rise to additional contributions to the structure functions. These higher twist terms are classified according to their $Q^{2}$ dependence $1 / Q^{2 n}$, where $n=0$ is leading twist (twist=2), $n=1$ is twist=4, etc. They are usually only important at high $x$ [15] although higher twist effects at very low $x$ have been considered recently [16].

- Nuclear binding effects. Fixed target DIS experiments obtain information on the $d$ density by scattering off a deuteron target. Theoretically the deuteron is often treated as a free neutron and proton; binding effects are neglected. Recently much work has been done in determining more realistic models of the deuteron. For more details see Sec. 3 .

Estimates of these uncertainties may be obtained by repeating several fits varying the conditions of each fit in order to ascertain the influence of the assumptions. In the following such a procedure is described with the aim of estimating uncertainties in the region of high $x$ and high $Q^{2}$, relevant to the new HERA data.

\subsection{Next-to-Leading Order QCD Fit of NC Data}

The DGLAP evolution equations [17] are solved in the next-to-leading order (NLO) $\overline{M S}$ factorisation scheme using the program QCDNUM [18]. To ensure that the perturbative approximation is valid a starting scale of $Q_{0}^{2}=4 \mathrm{GeV}^{2}$ is taken at which four parton densities are parameterized. These are the up and down valence quarks $\left(x u_{v}\right.$ and $\left.x d_{v}\right)$, the gluon $(x g)$, and the sea quark distribution $(x \mathrm{~S})$. The sea quark density is assumed to have equal components from quarks and anti-quarks and is defined as $x \mathrm{~S}=2 \mathrm{x}(\overline{\mathrm{u}}+\overline{\mathrm{d}}+\overline{\mathrm{s}}+\overline{\mathrm{c}})$ at $Q_{0}^{2}$ where $\bar{s}=\bar{u} / 2$ and $\bar{d}=\bar{u}$. Since the kinematic region of interest is at high $Q^{2}$, a massless approach for heavy quarks is used. The $x b$ density is evolved from zero at a $Q^{2}$ threshold defined such that $x b\left(x, Q^{2}\right)=0$ for $Q^{2}<(5)^{2} \mathrm{GeV}^{2}$ and the charm component is normalised to $2 \%$ of the sea quark density at $Q^{2}=Q_{0}^{2}$, which gives a good description of the $\mathrm{H} 1$ measurements [19] of $F_{2}{ }^{c \bar{c}}$, the charm induced structure function.

The functional forms of the parton densities at $Q_{0}^{2}$ are as follows:

$$
\begin{aligned}
& x u_{v}\left(x, Q_{0}^{2}\right)=A_{u} x^{B_{u}}(1-x)^{C_{u}}\left(1+D_{u} x+E_{u} \sqrt{x}\right) \\
& x d_{v}\left(x, Q_{0}^{2}\right)=A_{d} x^{B_{d}}(1-x)^{C_{d}}\left(1+D_{d} x+E_{d} \sqrt{x}\right) \\
& x g\left(x, Q_{0}^{2}\right)=A_{g} x^{B_{g}}(1-x)^{C_{g}}\left(1+D_{g} x+E_{g} \sqrt{x}\right)
\end{aligned}
$$




$$
x \mathrm{~S}\left(\mathrm{x}, \mathrm{Q}_{0}^{2}\right)=A_{S} x^{B_{S}}(1-x)^{C_{S}}\left(1+D_{S} x+E_{S} \sqrt{x}\right)
$$

The parameters $A_{u}$, and $A_{d}$ are determined by applying the valence counting rules and $A_{g}$ is determined in terms of the other normalization parameters through the momentum sum rule. The strong coupling constant, $\alpha_{s}$, was fixed at the value $\alpha_{s}\left(M_{Z}^{2}\right)=0.118$.

Measurements of $F_{2}$ from NMC [20] and BCDMS [21] on both proton and deuteron targets were used in the fit. In addition the $F_{2}$ data from $\mathrm{H} 1$ [5] were included for $Q^{2} \leq 120 \mathrm{GeV}^{2}$. Since there is no data to constrain the low $x$ behaviour of valence quarks the parameters $B_{u}$ and $B_{d}$ were fixed in the fits to the values given in the MRSR2 parameterization. The influence of higher twist effects and deuteron binding effects was reduced by requiring the data to have $Q^{2}>10 \mathrm{GeV}^{2}, W^{2} \geq 20 \mathrm{GeV}^{2}$ as well as $Q^{2} \geq 20 \mathrm{GeV}^{2}$ for $x \geq 0.5$. The fixed target data were corrected for target mass corrections using the Georgi-Politzer approach [14], and deuteron binding effects as described in [22]. Data with $x>0.7$ were discarded, since in this region target mass corrections and deuteron binding effects are considered to be too large for reliable correction. The normalizations of all data sets were left free within the quoted luminosity uncertainty. The fits were performed using the program MINUIT [23] which minimised the $\chi^{2}$ defined with statistical and uncorrelated systematic errors added in quadrature. Several fits were performed varying in turn the input assumptions on the fit.

- $\alpha_{s}$ was varied by \pm 0.003 at the scale $M_{Z}^{2}$.

- The influence of remaining higher twist effects was determined by increasing the $Q^{2}$ cut from $10 \mathrm{GeV}^{2}$ to $15 \mathrm{GeV}^{2}$.

- An uncertainty on the charm density was obtained by varying the the charm momentum fraction by factors of 2 .

- The strange component of the sea was varied by $\pm 25 \%$.

- No deuteron corrections were applied to the data.

The results of the fit are shown in the form of the reduced NC cross section (this term is defined in Ref. [24]) in Fig 1. Note that the uncertainty comes purely from the variation in the fitted PDFs, all electroweak parameters have been set to standard values. The total uncertainty on the $\mathrm{NC}$ cross-section is at the level of $1 \%$ increasing to $5 \%$ at $x=0.65$. This increase is largely due to the $\alpha_{s}$ variation and the $Q_{\min }^{2}$ cut applied to the data. The variation of the quark sea components has very little effect in this high $Q^{2}$ region, however, the effect of the deuteron corrections can be as large as 1\%. The present study has considered only 'theoretical' uncertainties. It is interesting to compare our results with the analysis of Botje [7], where experimental systematics are considered (as well as an $\alpha_{s}$ variation). Botje finds the uncertainty on $\tilde{\sigma}_{N C}$ to be $4 \%$ rising to $11 \%$ at $x=0.4$ and $5 \%$ to $8 \%$ at $x=0.6$. Thus it seems that at lower $Q^{2}$ the uncertainties are dominated by experimental errors, whereas at higher $Q^{2}$ and $x$ theoretical and experimental uncertainties are of similar size. 


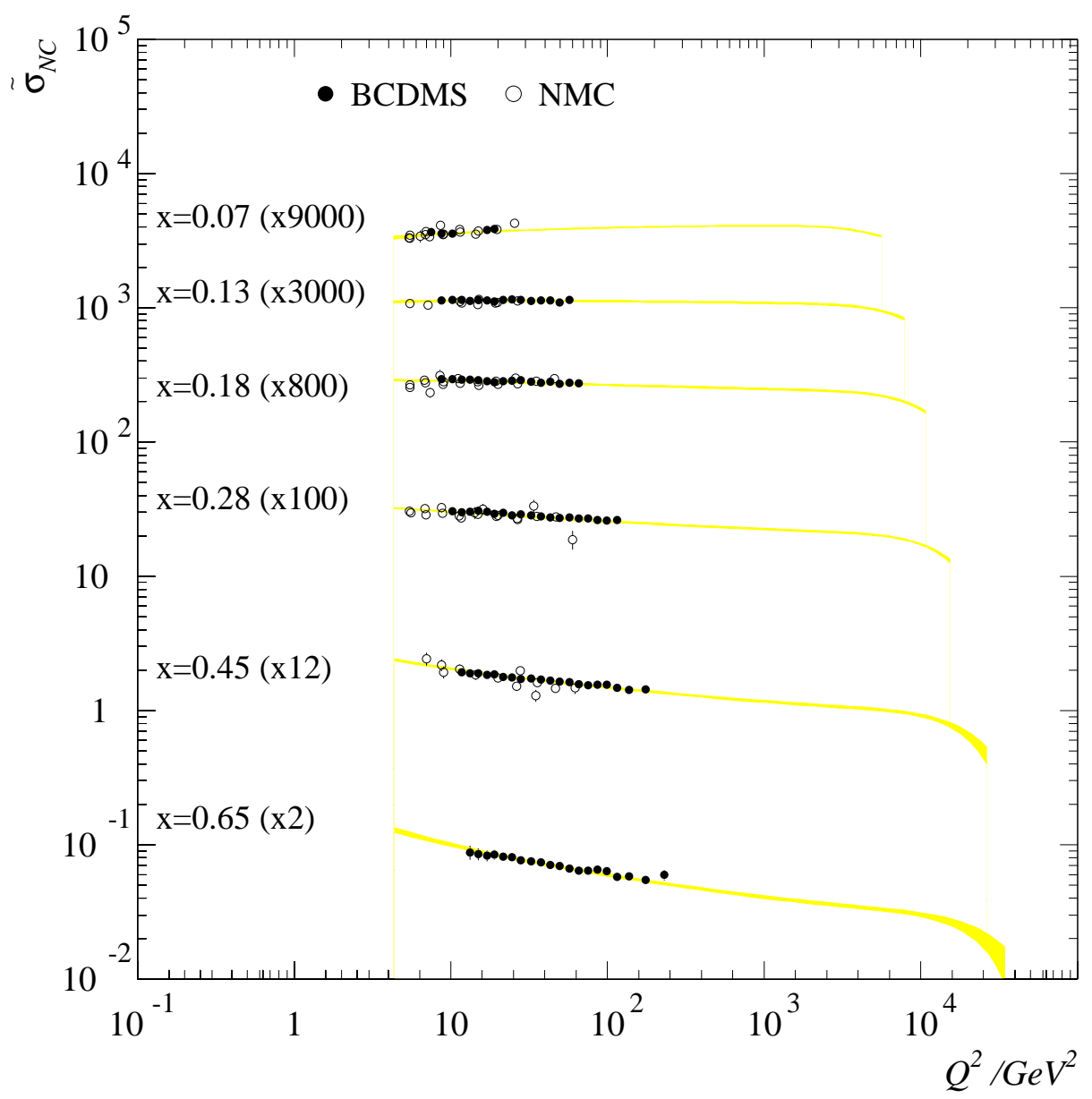

Figure 1. $\tilde{\sigma}_{N C}\left(e^{+}\right)$extrapolated to high $Q^{2}$. The band represents the uncertainties arising from variations in the input assumptions of the fit to NMC, BCDMS and H1 data.

\section{Parton Distributions, $d / u$, and HERA CC Data}

\subsection{Extraction of $d / u$ at high $x$}

The most accurate information about valence quarks originates from fixed target electro/muoproduction data on proton and deuteron targets. The $u$ valence quark distribution at high $x$ is relatively well constrained by the proton structure function $F_{2}^{p}$. However, the $d$ valence quark at high $x$ is constrained by the neutron structure function $F_{2}^{n}$, which is actually extracted from deuteron data. Therefore, there is an uncertainty in the $d$ valence quark distribution from the corrections for nuclear binding effects in the deuteron. In past extractions of $F_{2}^{n}$ from deuteron data, only Fermi motion corrections were considered, and other binding effects were assumed to be negligible. Recently, corrections for nuclear binding effects in the deuteron, $F_{2}^{d} / F_{2}^{n+p}$, have been 

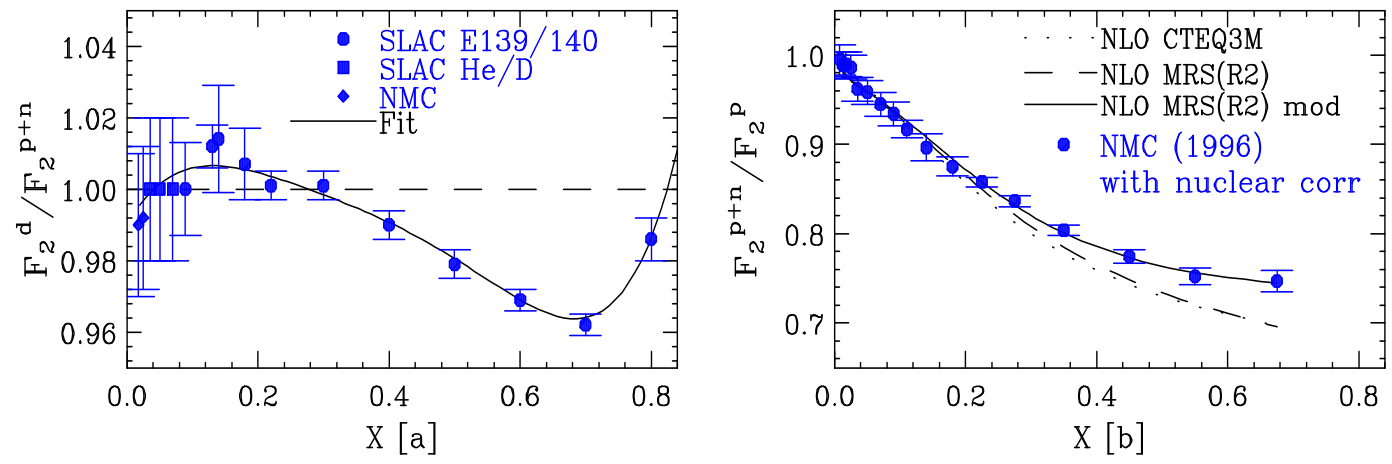

Figure 2. [a] The total correction for nuclear effects (binding and Fermi motion) in the deuteron, $F_{2}^{d} / F_{2}^{n+p}$, as a function of $x$, extracted from fits to the nuclear dependence of SLAC $F_{2}$ electron scattering data. [b] Comparison of NMC $F_{2}^{n+p} / F_{2}^{p}$ (corrected for nuclear effects) and the prediction in NLO using the MRS(R2) PDF with and without the proposed modification to the $d / u$ ratio. From ref. 25].

extracted empirically from fits to the nuclear dependence of electron scattering data from SLAC experiments E139/140 [26]. The empirical extraction uses a model proposed by Frankfurt and Strikman [22] in which all binding effects in the deuteron and heavy nuclear targets are assumed to scale with the nuclear density. The correction extracted in this empirical way is also in agreement (for $x<0.75$ ) with recent theoretical calculations [27] of nuclear binding effects in the deuteron. The total correction for nuclear binding in the deuteron is about $4 \%$ at $x=0.7$ (shown in Fig. 2[a]), and in a direction which is opposite to that expected from the previous models which only included the Fermi motion effects.

The ratio $F_{2}^{d} / F_{2}^{p}$ is directly related to $d / u$. In leading order $\mathrm{QCD}, 2 F_{2}^{d} / F_{2}^{p}-1 \simeq$ $(1+4 d / u) /(4+d / u)$ at high $x$. Bodek and Yang 25 have recently performed an NLO analysis on the precise NMC $F_{2}^{d} / F_{2}^{p}$ data 28 taking deuteron binding corrections into account. The ratio $F_{2}^{p+n} / F_{2}^{p}$ is extracted by applying the nuclear binding correction $F_{2}^{d} / F_{2}^{n+p}$ to the $F_{2}^{d} / F_{2}^{p}$ data. As shown in Fig. 2[b], the standard PDF's do not describe this corrected $F_{2}^{p+n} / F_{2}^{p}$. Since the $u$ distribution is relatively well constrained, Bodek and Yang suggest a correction term to $d / u$ in the standard PDF's (as a function of $x$ ), which changes the $d$ distribution to fit the data. This correction term is parametrized as a simple quadratic form, $\delta(d / u)=(0.1 \pm 0.01)(x+1) x$ for the MRS(R2) PDF, where the corrected $d / u$ ratio is $(d / u)^{\prime}=(d / u)+\delta(d / u)$. Based on this correction, an MRS(R2)modified PDF is obtained, as shown in Fig 2[b]. The correction to other PDF's such as CTEQ3M is similar. Fig [3[a] illustrates the effect of this modification on the $d / u$ ratio, where we can clearly see that $d / u$ in the standard PDF's approaches 0 as $x \rightarrow 1$, whereas the modified $d / u$ ratio approaches $0.2 \pm 0.02$ as $x \rightarrow 1$, in agreement with a QCD prediction [29]. Information on $d / u$ which is free from nuclear effects may also be extracted from $\nu p / \bar{\nu} p$ data, but unfortunately this is rather inaccurate. Fig. 3[a] shows that the CDHSW 30 data favour the modified PDF's at high $x$. Further information on $d / u$, which is not affected by the corrections for nuclear effects in the deuteron, 

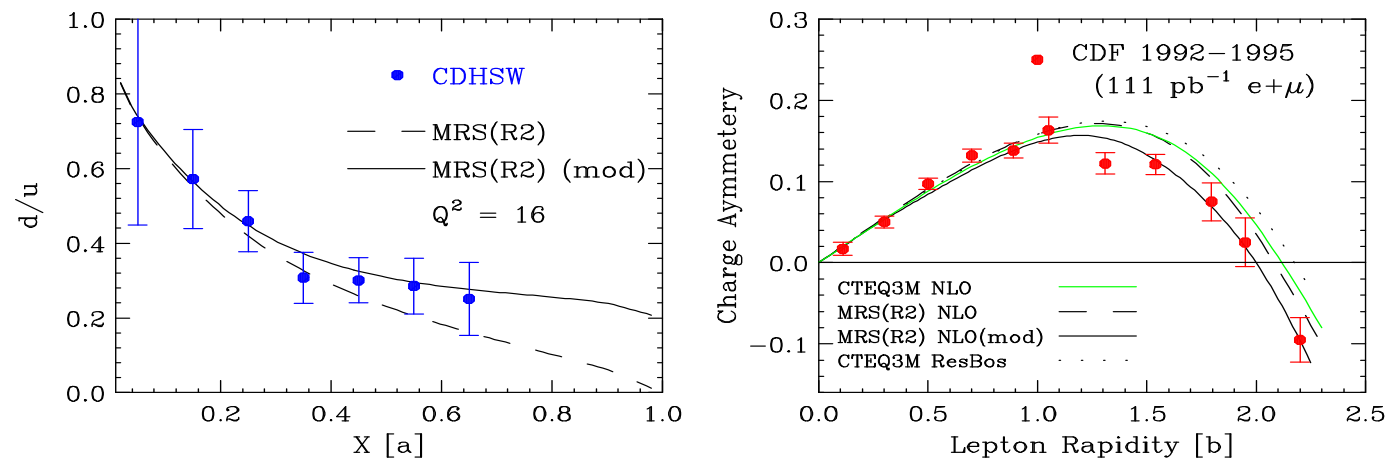

Figure 3. [a] The $d / u$ distributions at $Q^{2}=16 \mathrm{GeV}^{2}$ as a function of $x$ for the standard and modified MRS(R2) PDF compared to the CDHSW data. [b] Comparison of the CDF $W$ asymmetry data with NLO standard CTEQ3M, MRS(R2), and modified $\operatorname{MRS}(\mathrm{R} 2)$ as a function of the lepton rapidity. The standard CTEQ3M with a resummation calculation is also shown for comparison. From ref 25.
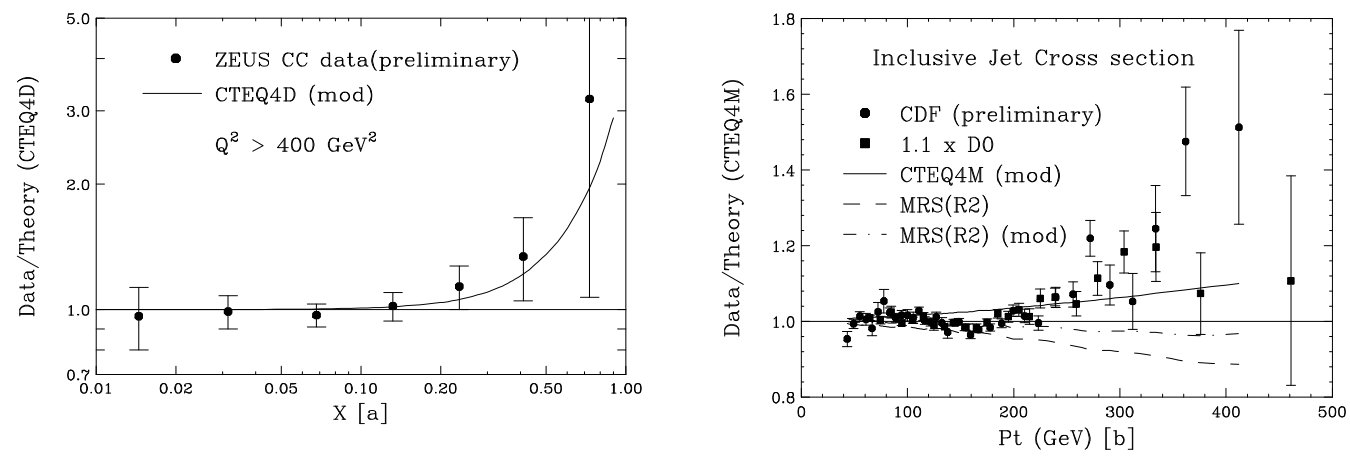

Figure 4. [a] The HERA charged current cross section data and [b] the CDF and D0 inclusive jet cross section data are compared with both standard and modified PDF's. From ref 25].

can be extracted from $W$ production data in hadron colliders. Fig $3[\mathrm{~b}]$ shows that the predicted $W$ asymmetry calculated with the DYRAD NLO QCD program using the modified PDF is in much better agreement with recent CDF data [31] at large rapidity than standard PDF's.

Clearly future $e^{+} / e^{-}$HERA CC data can provide new information on the $d / u$ ratio, free from nuclear effects. When the modified PDF at $Q^{2}=16 \mathrm{GeV}^{2}$ is evolved to $Q^{2}=10^{4}$ $\mathrm{GeV}^{2}$ using the DGLAP NLO equations, it is found that the modified $d$ distribution at $x=0.5$ is increased by about $40 \%$ in comparison to the standard $d$ distribution. Fig. $4[\mathrm{a}]$ shows the HERA $e^{+} \mathrm{CC}$ cross section data compared to the predictions of the CTEQ4D distributions both with and without the modification. One can easily see that an increased luminosity of such data will be crucial in deciding the need for any modification. Fig. [ [ [b] shows that the modified PDF's also lead to an increase of $10 \%$ in the QCD prediction for the production rate of very high $P_{T}$ jets [33] in hadron colliders. 

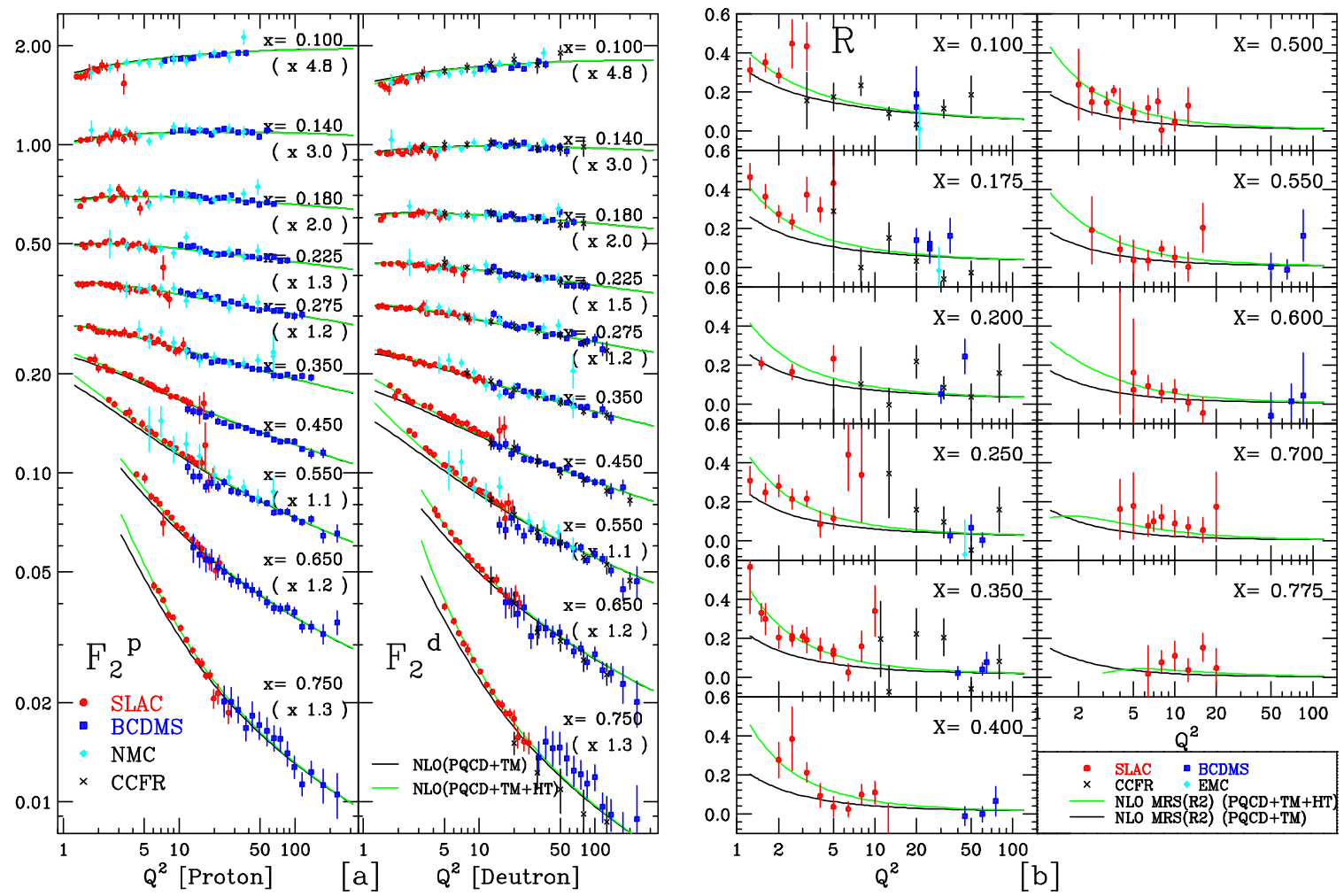

Figure 5. Comparison of the modified NLO MRS(R2) PDF plus target mass and renormalon higher twist corrections, with low $Q^{2}$ data. [a] Comparison of $F_{2}$ and NLO prediction with and without higher twist contributions. [b] Comparison of $R$ and NLO prediction with and without the higher twist contributions. From ref. 25.

\subsection{Target mass, higher twist and PDFs at very high $x$}

Since all the standard PDF's, including the modified versions, are fit to data with $x$ less than 0.75 , Bodek and Yang also investigate the validity of the modified MRS(R2) at very high $x(0.75<x<0.98)$ by comparing to $F_{2}^{p}$ data at SLAC. Although the SLAC data at very high $x$ are at reasonable values of $Q^{2}\left(7<Q^{2}<31 \mathrm{GeV}^{2}\right)$, they are in a region in which non-perturbative effects such as target mass and higher twist are very large. The Georgi-Politzer calculation is used [14 for the target mass corrections (TM). These involve using the scaling variable $\xi=2 x /\left(1+\sqrt{1+4 M^{2} x^{2} / Q^{2}}\right)$ instead of $x$. Since a complete calculation of higher twist effects is not available, data at lower $Q^{2}$ $\left(1<Q^{2}<7 \mathrm{GeV}^{2}\right.$ and $\left.x<0.75\right)$ are used to obtain information on the size of these terms. Two approaches are used: an empirical method and the renormalon model [34], full details of the formalisms are given in 25$]$. Both approaches describe the data well.

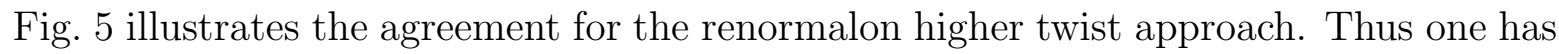
confidence in extrapolating these approaches to investigate the very high $x$ region.

There is a wealth of SLAC data 35 in the region up to $x=0.98$ and intermediate $Q^{2}\left(7<Q^{2}<31 \mathrm{GeV}^{2}\right)$. Previous PDF fits have not used these data. The data for $0.9>x>0.75$ is in the DIS region, and the data for $x>0.9$ is in the resonance region. It is worthwhile investigating the resonance region because duality arguments [36] 

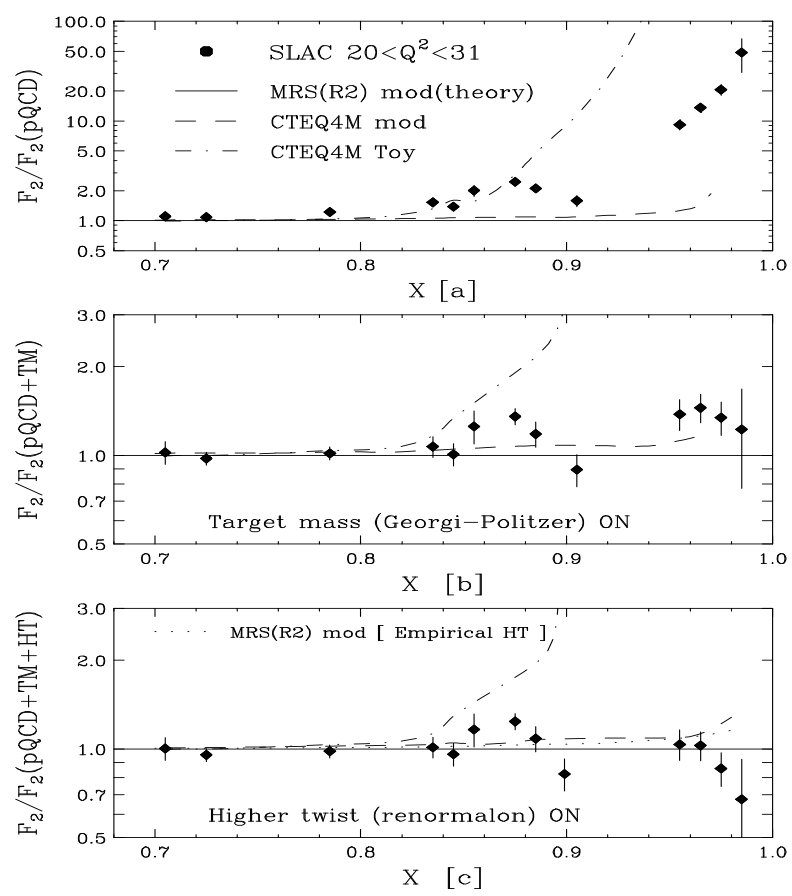

Figure 6. Comparison of SLAC $F_{2}^{p}$ data with the predictions of the modified MRS(R2), CTEQ4M and the CTEQ toy model at high $x$ and $20<Q^{2}<31 \mathrm{GeV}^{2}$. [a] Ratio to pQCD, [b] ratio to pQCD with TM effects, and [c] ratio to pQCD with TM and higher twist effects. From ref. 25].

indicate that the average behaviour of the resonances and elastic peak should follow the DIS scaling limit curve. Fig. 6 shows the ratio of the SLAC data to the predictions of the modified MRS(R2) at $Q^{2}$ values $\left(21<Q^{2}<30 \mathrm{GeV}^{2}\right)$ where the elastic contribution is negligible. Fig $6[$ c] shows that, with the inclusion of target mass and renormalon higher twist effects, the very high $x$ data from SLAC is remarkably well described by the modified MRS(R2) right up to $x=0.98$. Fig $6[\mathrm{c}]$ also illustrates that a good description is achieved using the empirical estimate of higher twist effects. Fig. 6 also shows that the CTEQ Toy model (with an additional $0.5 \%$ component of $u$ quarks beyond $x>0.75$ ) overestimates the SLAC data by a factor of three at $x=0.9$ (DIS region). From these comparisons, it is found that the SLAC $F_{2}$ data do not support the CTEQ Toy model which proposed an additional $u$ quark contribution at high $x$ as an explanation of the initial HERA high $Q^{2}$ anomaly and the CDF high- $P_{t}$ jet excess.

Thus it seems that there is a need for modification of current standard PDFs at high $x$, but this conclusion is dependent on the correctness of deuteron binding calculations. Future precise charged current data from HERA will provide vital information on valence quark densities at high $x$ and high $Q^{2}$, free from this uncertainty.

\section{Electroweak Radiative Corrections}

Electroweak radiative corrections of order $O(\alpha)$ have to be taken into account if a precision of $1 \%$ is to be achieved in physics analyses at HERA. Pure QED corrections 
contain contributions which are enhanced by logarithmic factors like $\log \left(Q^{2} / m_{e}^{2}\right)$. In addition, radiation of photons can shift kinematic variables from very large to very small values inducing additional enhancement factors for NC scattering. Furthermore, purely weak one-loop corrections will eventually be important for a measurement of $M_{W}$ with a precision of $O(100 \mathrm{MeV})$.

As discussed in much detail in the literature [37], the differential cross section for $\mathrm{NC}$ scattering when measured in terms of electron variables receives QED corrections which are large and negative at small $y_{e}$ and large $x_{e}$, whereas at large $y_{e}$ and small $x_{e}$ large positive corrections may reach the level of $100 \%$. These corrections can be reduced, sometimes considerably, with measurements based on hadronic or mixed kinematic variables. Self energy corrections (the running of $\alpha\left(Q^{2}\right)$ ) are of order $10 \%$ and, finally, purely weak corrections are negligible at low $Q^{2}$, but increase to the level of a few per cent at very large $Q^{2}$.

In the following we give a more detailed discussion of electroweak radiative corrections to the charged current process [38, 39, 40], firstly since their treatment is more involved and has been less well described in the literature than the NC case; secondly because the implementations in available numerical programs appear to have larger uncertainties than the corresponding $\mathrm{NC}$ codes.

Fig. 7 shows the complete set of electroweak one-loop Feynman diagrams contributing to $e q_{f} \rightarrow \nu q_{f^{\prime}}$ scattering. The list comprises corrections to the $e \nu W$ and $q_{f} q_{f^{\prime}} W$ vertices, self energy corrections to the external lines, graphs for the $W$ self energy and box diagrams. Diagrams with an additional photon in the loop gives rise to infrared divergent contributions. They can be regularized, for example by using a finite mass for the photon, and cancel against similarly infrared divergent contributions from radiative CC scattering $e q_{f} \rightarrow \nu q_{f^{\prime}} \gamma$ (see Fig. 8) when calculating the cross section.

The calculation is more complicated than the one for NC scattering since for the $\mathrm{CC}$ case there is no simple enough subset of Feynman diagrams which is gauge invariant. In particular, all four diagrams of Fig. 8 describing the emission of a photon from the incoming lepton (a), from the incoming (b) and outgoing quark (c) and from the internal $W(\mathrm{~d})$ are necessary to obtain a physically meaningful result.

Internal fermion propagators in the diagrams of Fig. 8 give rise to terms with pole factors which become large when the photon is collinear with one of the external charged fermions. The differential cross section for radiative $\mathrm{CC}$ scattering can thus be written as

$$
\left.\frac{d^{5} \sigma}{d x d Q^{2} d^{3} k}\right|_{e q \rightarrow \nu q^{\prime} \gamma}=\frac{A_{l}}{k l}+\frac{A_{q}}{k q}+\frac{A_{q^{\prime}}}{k q^{\prime}}+\text { non }- \text { pole terms }
$$

where the notation shown in Fig. 8(a) for the particle's 4-momenta was used. This shows that the numerical calculation of the $\gamma$-inclusive cross section has to cope with nearly-divergent behavior in different phase space regions. Note that the separation into pole factors in Eq. 11 is not unique: finite (i.e. non-pole) terms can be shifted freely between the three contributions. A specific separation of the radiative cross section like that given in Eq. 11 is needed for the construction of a well-behaved numerical 

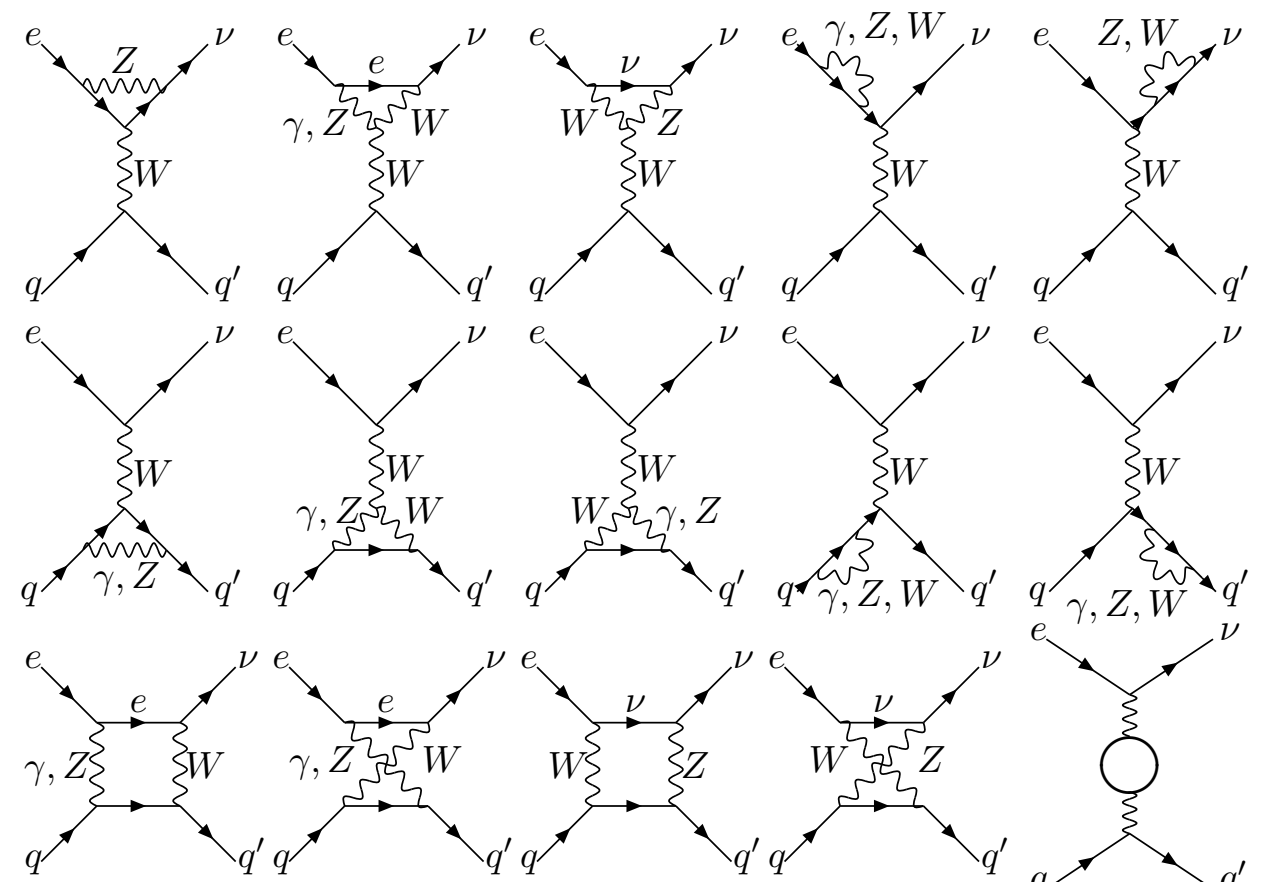

Figure 7. One-loop Feynman diagrams for $e q \rightarrow \nu q^{\prime}$ scattering.

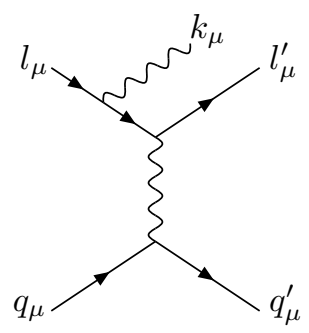

(a)

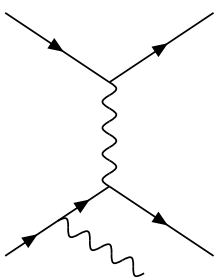

(b)

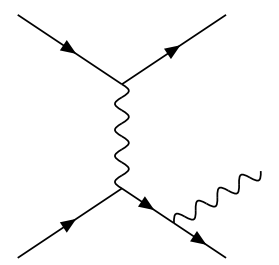

(c)

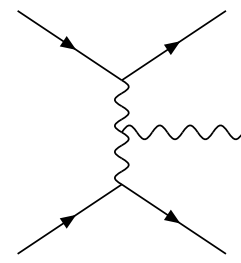

(d)

Figure 8. Feynman diagrams for radiative charged current scattering $e q \rightarrow \nu q^{\prime} \gamma$ and notation of 4-momenta for external particles.

algorithm, but the individual contributions do not have a well-defined physical meaning when taken separately.

To obtain a gauge invariant, i.e. physically meaningful separation, one can organize the terms contributing to the complete cross section according to their dependence on the electric charge of the incoming particles. Replacing the charge of the $W^{ \pm}$by that of the incoming lepton, $e_{l}=e_{W}$, and that of the final quark $q_{f^{\prime}}$ by that of the incoming quark $q_{f}$ and the lepton, $e_{f^{\prime}}=e_{f}+e_{l}$, one can write

$$
\left.\frac{d^{5} \sigma}{d x d Q^{2} d^{3} k}\right|_{e q \rightarrow \nu q^{\prime} \gamma}=e_{l}^{2} I_{\mathrm{lep}}+e_{l} e_{f} I_{\mathrm{int}}+e_{f}^{2} I_{\mathrm{qua}} .
$$

The individual parts in this separation are gauge invariant and due to their association to fermion charges it is justified to call them "leptonic", "interference", and "quarkonic", as indicated by the subscripts in Eq. 12. A closer inspection of the diagrams in Fig. 8 shows that $I_{\text {lep }}$ contains pole terms $\propto 1 / k l$ and $1 / k q^{\prime}$, whereas $I_{\text {qua }}$ contains terms $\propto 1 / k q$ and $1 / k q^{\prime}$. The interference contribution $I_{\text {int }}$ receives contributions from all three pole factors 
$1 / k l, 1 / k q$ and $1 / k q^{\prime}$. After integration over the photon momentum, the pole factors give rise to logarithms of the corresponding fermion masses: $\log \left(Q^{2} / m_{e}^{2}\right), \log \left(Q^{2} / m_{q_{f}}^{2}\right)$ and $\log \left(Q^{2} / m_{q_{f^{\prime}}}^{2}\right)$. Here we assume that collinear singularities are regularized by introducing finite masses for quarks.

The virtual corrections from Fig. 7 can be separated in the same way. After combining real and virtual corrections, not only do infrared divergent contributions cancel (allowing the removal of the regularization by the finite photon mass), but also many mass logarithms disappear in the complete final result: writing the complete $O(\alpha)$-corrected cross section similarly to Eq. 12:

$$
\left.\frac{d^{5} \sigma}{d x d Q^{2} d^{3} k}\right|_{e q \rightarrow \nu q^{\prime}(+\gamma)}=e_{l}^{2} J_{\text {lep }}+e_{l} e_{f} J_{\text {int }}+e_{f}^{2} J_{\text {qua }},
$$

one finds that $i$ ) all logarithms related to the final quark disappear, i.e. $J_{\text {lep }}$ contains $\log \left(Q^{2} / m_{e}^{2}\right)$ terms only, $J_{\text {qua }}$ contains $\log \left(Q^{2} / m_{q_{f}}^{2}\right)$ terms only and $\left.i i\right)$ all mass logarithms cancel in $J_{\text {int }}$, i.e. the interference contribution contains no mass singularities. Therefore the effect of photonic corrections on the cross section for CC scattering can be summarized as follows:

- Leptonic contributions proportional to $e_{l}^{2}$ contain terms which are enhanced by the large $\operatorname{logarithm} \log \left(Q^{2} / m_{e}^{2}\right)$. This contribution has a dependence on kinematic variables which is very similar to the $\mathrm{NC}$ case except that the enhancement at large $y_{e}$ is much less pronounced because of the much weaker dependence of the CC Born cross section on $Q^{2}$.

- Interference contributions are of order $O(\alpha / \pi)$ without logarithmic enhancement.

- Quarkonic contributions contain uncancelled $\log \left(Q^{2} / m_{q_{f}}^{2}\right)$ terms. These mass singularities have the same structure as those appearing in a calculation of $O\left(\alpha_{\mathrm{s}}\right)$ QCD corrections due to the emission of gluons from incoming and scattered quarks (in dimensional regularization one would find the same $1 / \epsilon$ divergences apart from differing charge factors: $\alpha e_{f}^{2}$ instead of $\alpha_{\mathrm{s}} C_{F}$ ). As with QCD corrections, the mass singularities due to QED corrections can be factorized and absorbed into renormalized parton distribution functions. Thereby the final results do not depend any more on a regularization parameter (like the quark mass), but the $Q^{2}$-evolution equations for parton distribution functions receive an additional QED term:

$$
\begin{gathered}
Q^{2} \frac{d}{d Q^{2}} q_{f}\left(x, Q^{2}\right)=\frac{\alpha_{\mathrm{s}}\left(Q^{2}\right)}{2 \pi} \int_{0}^{1} \frac{d z}{z}\left(C_{F} P_{f / f}(z) q_{f}\left(\frac{x}{z}, Q^{2}\right)+P_{f / g}(z) g\left(\frac{x}{z}, Q^{2}\right)\right) \\
+\frac{\alpha\left(Q^{2}\right)}{2 \pi} e_{f}^{2} \int_{0}^{1} \frac{d z}{z} P_{f / f}(z) q_{f}\left(\frac{x}{z}, Q^{2}\right) .
\end{gathered}
$$

Numerical estimates [41] show that such effects reach the level of $1 \%$ only at very large $x$ and $Q^{2}$.

One should note that the available numerical programs either simply ignore the quarkonic contributions or suppress them by using a relatively large value for the quark mass (of the order of several $\mathrm{GeV}$ ). Another level of approximation justified by the 
above discussion is to neglect the interference contribution. Numerical results for the individual contributions have been given in [38, 40.

Having separated the purely photonic corrections as described above, there still remain purely weak contributions (most prominently, but not exclusively, due to the $W$ self energy) which can be combined into over-all form factors [38, 42]. For electron scattering with unpolarized beams Eq. 9 becomes:

$$
\begin{aligned}
\left.\frac{d \sigma}{d x d Q^{2}}\right|_{e^{-} p} ^{C C}= & \frac{G_{\mu}^{2}}{2 \pi}\left(\frac{M_{W}^{2}}{Q^{2}+M_{W}^{2}}\right)^{2}\left\{\left(\rho_{C C}^{e u}\left(x, Q^{2}\right)\right)^{2} \sum_{f=u, c} q_{f}\left(x, Q^{2}\right)\right. \\
& \left.+(1-y)^{2}\left(\rho_{C C}^{e d}\left(x, Q^{2}\right)\right)^{2} \sum_{f=d, s} \bar{q}_{f}\left(x, Q^{2}\right)\right\} .
\end{aligned}
$$

The form factors $\rho_{C C}^{e f}\left(x, Q^{2}\right)$ depend on $Q^{2}$ and $x$ as well as on the type of the scattered quark (due to the presence of box diagrams). The above expression assumes that the $\mathrm{CC}$ cross section is normalized with the help of the $\mu$-decay constant $G_{\mu}$.

The cross section also depends explicitly on the $W$ boson mass and, through the form factors $\rho_{C C}^{e f}$, on all other parameters of the electroweak standard model.

\subsection{Choice of independent parameters}

One possible set of independent parameters is $\alpha, m_{t}$ (the top mass), $M_{H}$ (the Higgs mass), $M_{W}, M_{Z}$ (in addition, there are light fermion masses and CKM matrix elements, which we do not consider). A special role is played by $\alpha$ which is kept fixed since it is well-measured and is a parameter of QED which is embedded in the full electroweak theory. The Higgs mass is also special: because of the weak dependence on $M_{H}$ in present day experiments, one can keep it as an external parameter and investigate the effect of changing it separately (see Sec. 5). Thus the essential parameters are $M_{W}, M_{Z}$ and $m_{t}$, since $G_{\mu}$ is related to these parameters by the SM expression given in Eq. 16 .

We consider the following two possible scenarios:

- Choose $G_{\mu}, M_{W}$ and $M_{Z}$ as independent parameters. Then $m_{t}$ is a prediction and a specific choice of values for $G_{\mu}, M_{W}$ and $M_{Z}$ will predict a value for $m_{t}$ (possibly in disagreement with experimental results). Fixing $G_{\mu}$ and $M_{Z}$ to their measured values (from $\beta$ decay and LEP experiments, respectively) the CC cross section depends on $M_{W}$ and a measurement at HERA can be interpreted as a measurement of the $W$ mass. Note that this assumes the validity of the Standard Model and the measurement is therefore not a direct $W$-mass measurement. (This is also true for scenario 2). Alternatively, keeping $M_{W}$ and $M_{Z}$ fixed, the charged current cross section could be interpreted as a measurement of $G_{\mu}$. This is interesting since it allows a direct comparison of the high- $Q^{2}$ experiments at HERA with the low-energy measurement of $\mu$ decay

- Choose $M_{W}, M_{Z}$ and $m_{t}$ as independent parameters (on-shell scheme). Then $G_{\mu}$ 
is a prediction of the theory given by

$$
G_{\mu}=\frac{\pi \alpha}{\sqrt{2} s_{\mathrm{w}}^{2} M_{W}^{2}} \frac{1}{1-\Delta r} \quad \text { with } \quad s_{\mathrm{w}}^{2}=1-\frac{M_{W}^{2}}{M_{Z}^{2}}
$$

and $\Delta r$, which embodies the radiative corrections to the $\mu$-decay, is a function of $M_{W}, M_{Z}, m_{t}$ (and $\alpha, M_{H}$, of course). $\Delta r$ has to be taken into account in a consistent $O(\alpha)$ prescription and should be combined with the other $O(\alpha)$ corrections in the $\mathrm{CC}$ cross section formula, i.e. replacing

$$
\rho_{C C}^{e f} \longrightarrow \frac{\rho_{C C}^{e f}}{(1-\Delta r)^{2}}=\hat{\rho}_{C C}^{e f} .
$$

Because of the inclusion of $\Delta r$ the new form factors have a strong dependence on $m_{t}$ since

$$
\Delta r=\Delta \alpha-\frac{c_{w}^{2}}{s_{\mathrm{w}}^{2}} \Delta \bar{\rho}+\Delta r^{\mathrm{rem}} \quad \text { with } \quad \Delta \bar{\rho}=\frac{3 G_{\mu} m_{t}^{2}}{8 \pi^{2} \sqrt{2}} .
$$

Furthermore the dependence of the CC cross section on $M_{W}$ is much stronger due to the replacement

$$
\left(\frac{m_{W}^{2}}{Q^{2}+m_{W}^{2}}\right)^{2} \longrightarrow\left(\frac{1}{1-m_{W}^{2} / m_{Z}^{2}} \frac{1}{Q^{2}+m_{W}^{2}}\right)^{2} .
$$

Applied to the situation at HERA, keeping $M_{Z}$ and $m_{t}$ at their experimental values, the measurement of the $\mathrm{CC}$ cross section can once more be interpreted as an indirect measurement of $M_{W}$ 43, 44 which is, through Eq. 16, equivalent to a measurement of $G_{\mu}$, however in a process at large momentum transfer.

Both schemes are theoretically equivalent, a specific choice should be motivated by the aim of the analysis. We repeat: the above discussion focussed on the essential parameters $M_{W}, M_{Z}, m_{t}$, but, in addition, a weak dependence on $M_{H}$ is always present, both in $\Delta r$ as well as in the form factors $\rho_{C C}^{e f}$, this is quantified further in Sec. 5

It might be instructive to see how the situation simplifies in the case when radiative corrections are neglected altogether. Then $m_{t}$ and $M_{H}$ do not play any role for CC DIS. In scenario 1 where $G_{\mu}$ and $M_{W}$ are considered as independent parameters, $M_{Z}$ is also irrelevant. With fixed $G_{\mu}$, the measured cross section can be fitted by varying $M_{W}$. The resulting value has been called a 'propagator mass' in previous experimental literature since it is due to the dependence of the cross section on $M_{W}$ via the propagator only. Keeping $M_{W}$ fixed leaves no possibility to adjust the $Q^{2}$ dependence, and then the measurement only determines the normalization (in terms of $G_{\mu}$ ) of the cross section. Scenario 2, with fixed $M_{Z}$, allows us to combine the sensitivity to the propagator and to the normalization in one common parameter, i.e. the $W$ mass, as made explicit in Eq. 19. In Sec. 5 we consider both scenarios.

\subsection{Numerical results and discussion of uncertainties}

The presently available numerical programs for the calculation of the CC cross section do not all take into account the complete set of one-loop and one-photon corrections 


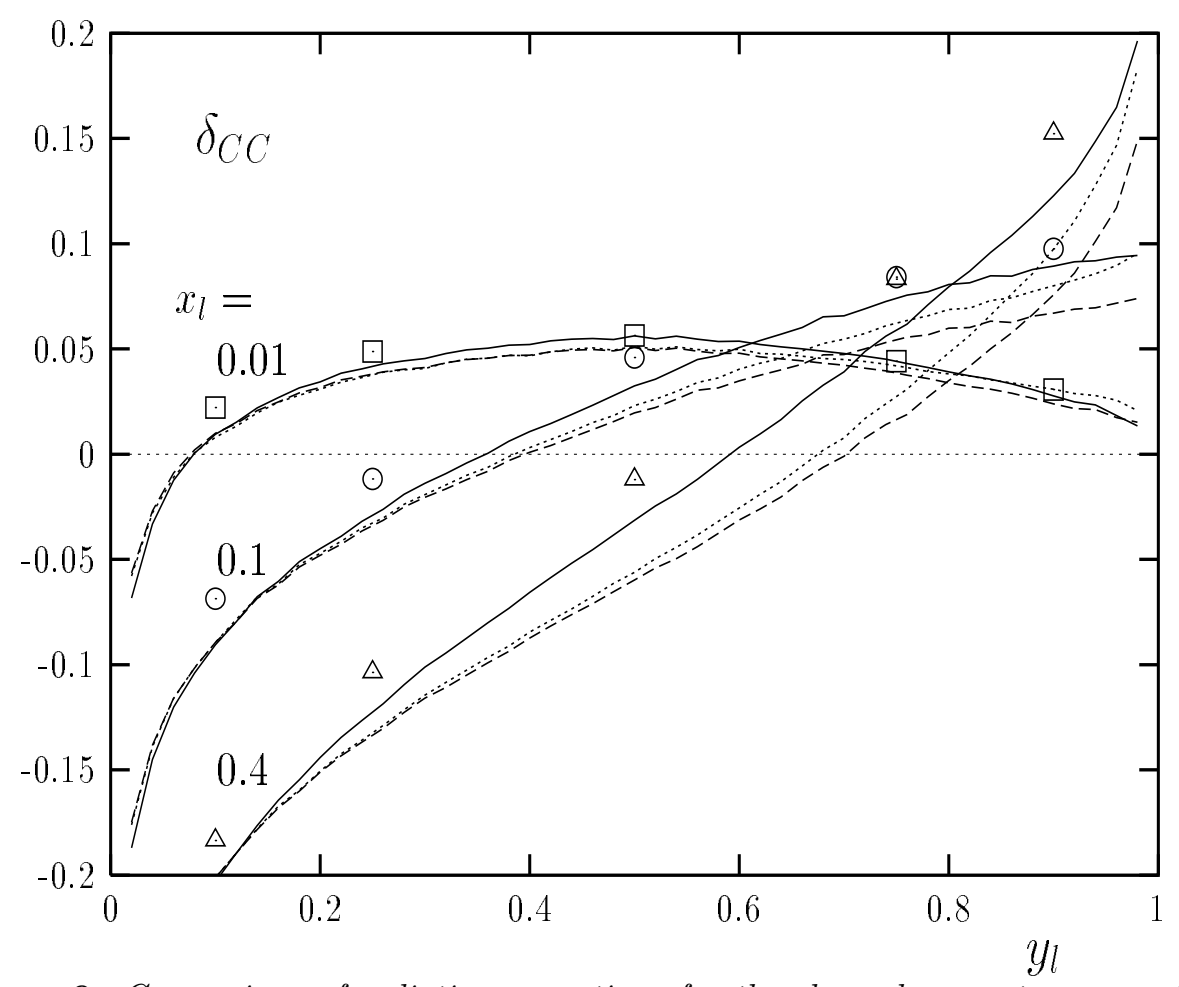

Figure 9. Comparison of radiative corrections for the charged current cross section, $\delta_{C C}=d \sigma /\left.d x_{l} d y_{l}\right|_{O(\alpha)} / d \sigma /\left.d x_{l} d y_{l}\right|_{\text {Born }}-1$, as a function of $y_{l}$ for fixed values of $x_{l}$. The full curves include the complete QED and weak corrections, the dashed curves are without purely weak corrections and the dotted lines show the leptonic QED corrections only (all from epcctot). Open symbols are obtained from DJANGOH and include leptonic QED and purely weak corrections.

described above. Two programs with the complete set of $O(\alpha)$ corrections (DISEPW [38 and epcctot [45]) have been compared in the HERA workshop [37] and found to agree well except in the phase space corners of small $x$, small $y$ or large $x$, all $y$. These programs are, however, not very well-suited for an application to realistic experiments since they do not allow to apply experimental cuts and, secondly, since they are restricted to the use of leptonic variables whereas experiments have to use hadronic variables. The Monte Carlo event generator HERACLES/DJANGOH [46] circumvents these two restrictions, however, it has the $\mathrm{CC}$ radiative corrections implemented in an approximation where photonic radiative corrections from lepton-quark interference and from quark radiation are neglected. Theoretically this is motivated to the extent that an accuracy of $1 \%$ is sufficient, as discussed above.

Fig. 9 shows a comparison of results obtained with the most recent version of DJANGOH with the complete $O(\alpha)$ results from epcctot for lepton variables. At small values of $x_{e}$, the comparison is satisfactory, but at larger values of $x_{e}$, the Monte Carlo results lie above those from epcctot. Here improvements are still needed. More detailed comparisons, in particular between DJANGOH and HECTOR [47, are presently being performed [48].

Apart from the approximations in available program implementations, there are additional intrinsic uncertainties of predictions for the $\mathrm{CC}$ cross section. First, a 
theoretically consistent treatment of quarkonic radiation as described above is not available yet. The uncertainty from this source is most likely below $1 \%$, and is thus negligible except at very large $Q^{2}$ [1]. Secondly, higher-order corrections are to be expected, first of all from multi-photon effects, but also from combined QED-QCD corrections of $O\left(\alpha \alpha_{\mathrm{s}}\right)$ and possibly from two-loop weak corrections. Multi-photon effects due to soft photons can be treated by exponentiation. This effect can be taken into account only in epcctot or HECTOR. It is expected to be numerically significant at low $y_{e}$, low $x_{e}\left(O(2 \%)\right.$ for $\left.x_{e}<0.1\right)$ and very large $x_{e}\left(O(10 \%)\right.$ for $\left.x_{e}>0.9\right)$ [40], i.e. close to the phase space boundaries. Two-photon contributions of $O\left(\alpha^{2}\right)$ in the leading logarithmic approximation are also known and can be calculated with the help of HECTOR but no numerical evaluation of this contribution has been performed for CC scattering. $O\left(\alpha \alpha_{\mathrm{s}}\right)$ corrections are not expected to become important. An indication of this comes from the fact that in NC scattering the larger $O\left(\alpha_{\mathrm{s}}\right)$ correction to $F_{3}$ stays below $1.5 \%$, for values of $x$ and $Q^{2}$ relevant at HERA. Two-loop weak contributions are also not expected to be important for the cross section itself. However, two-loop effects can affect the value of $M_{W}$ at the level of several tens of $\mathrm{MeV}$ when determined from $G_{\mu}$ via Eq. 16. The dominating higher-than one-loop effects to the $G_{\mu}-M_{W}$ relation are implemented in all availabe programs, but not all of the many small effects discussed above have been taken into account while the dominating source of uncertainty is still the statistical error.

\section{The Determination of $M_{W}$ in Charged Current Deep Inelatic Scattering}

\subsection{Introduction}

Recently the ZEUS and H1 experiments at the HERA positron-proton collider announced preliminary measurements of the single and double differential cross sections for the $\mathrm{CC}$ reaction $e^{+} P \rightarrow \bar{\nu}_{e} X$ [49, 50]. The measurements were made using data sets corresponding to a luminosity of $\sim 40 \mathrm{pb}^{-1}$ per experiment. The CC data is now sufficiently precise to make it timely to re-examine the extent to which the HERA experiments can contribute to constraints on the electroweak sector of the SM. In the following the statistical precision with which the parameters $M_{W}$ or $G_{\mu}$ may be determined will be investigated. Hence, it is the dependence of the CC cross section on $G_{\mu}$ or $M_{W}$ which is of primary importance and small differences between different approaches to the calculation of the radiative corrections themselves will not be discussed. There is no attempt in what follows to discuss the experimental systematic uncertainties involved in measurements such as those proposed.

\subsection{Sensitivity of $C C D I S$ to $M_{W}$}

The Born cross-section for the reaction $e^{+} P \rightarrow \bar{\nu}_{e} X$ has been given in Eq. 8. In this expression the coupling of the $W$-boson to the fermions is contained in $G_{\mu}$ while the $W$-boson propagator contributes the factor $\left[M_{W}^{2} /\left(M_{W}^{2}+Q^{2}\right)\right]^{2}$. 
The mass of the exchanged boson can be determined from the $Q^{2}$ dependence of the CC cross section since the propagator factor $\left(\left[M_{W}^{2} /\left(M_{W}^{2}+Q^{2}\right)\right]^{2}\right)$ depends explicitly on $M_{W}$. This is the 'propagator' mass mentioned in Sec. 1 (scenario 1 with fixed $G_{\mu}$ ). Both the ZEUS and H1 collaborations have performed such a determination with the results 49, 50,

$$
\begin{aligned}
& M_{W}=78.6_{-2.4}^{+2.5}[\text { stat }]_{-3.0}^{+3.3}[\text { syst }]_{-1.5}^{+1.5}[\mathrm{PDF}] \mathrm{GeV} \quad(\text { ZEUS }), \\
& M_{W}=81.2_{-3.3}^{+3.3}[\text { stat }]_{-4.3}^{+4.3}[\text { syst }]_{-2.8}^{+2.8}[\mathrm{PDF}] \mathrm{GeV} \quad(\mathrm{H} 1) .
\end{aligned}
$$

The uncertainty on the value of $M_{W}$ arising from the PDFs is quoted separately above. This error is determined by performing the fit using a variety of PDFs (additionally, the error quoted by $\mathrm{H} 1$ includes a $4 \%$ error on the $\mathrm{CC}$ radiative corrections).

The data may also be used to determine $G_{\mu}$ or $M_{W}$ (assuming scenario 2). The precision with which $G_{\mu}$ can be determined is directly related to the precision with which the cross section is measured. The size of the $e^{+} P$ data set available to ZEUS and H1 yields $\sim 1000 \mathrm{CC}$ events per experiment. Hence the statistical uncertainty on such a direct determination of $G_{\mu}$ will be $\sim 1.7 \%$. Note that at HERA such a determination of $G_{\mu}$ will be performed at an effective $Q^{2}$ in excess of $\sim 400 \mathrm{GeV}^{2}$ and hence will be complementary to the precise measurement of $G_{\mu}$ obtained from muon decay where the momentum scale is set by the mass of the muon.

At $\mathcal{O}(\alpha)$ in the SM $G_{\mu}$ depends upon $M_{W}$ through Eq. 16. In the following we shall set $\alpha, M_{Z}$, and all fermion masses, other than $m_{t}$ to the values quoted in reference 51. As shown in Fig 10, $G_{\mu}$ depends strongly on $M_{W}$ and less strongly on the mass of the Higgs boson, $M_{H}$, or the mass of the top quark, $m_{t}$. Hence, within the context of the SM, the greatest sensitivity to $M_{W}$ may be obtained by combining the $M_{W}$ dependence of the propagator term in the CC cross section with the $M_{W}$ dependence of $G_{\mu}$. A naive propagation of the statistical error from a sample of $1000 \mathrm{CC}$ events leads to an error of $\sim 300 \mathrm{MeV}$ on the parameter $M_{W}$. More detailed numerical estimates are given in the next section. The figure shows the expected statistical error on a measurement of $G_{\mu}$. It can be seen that the variation of $G_{\mu}$ with $m_{t}$ or $M_{H}$ at fixed $M_{W}$ is negligible compared to the expected statistical error.

Before proceeding to numerical estimates of the precision with which $M_{W}$ or $G_{\mu}$ can be extracted from present CC data it is necessary to consider the dependence of the radiative corrections to the Born cross section (Eq. 8) on $M_{W}$. The radiative corrections to the Born cross section given in Eq. 15 may be formally expressed in terms of a correction factor, $\delta_{\mathrm{Rad}}$, defined by the equation

$$
\frac{d^{2} \sigma_{\mathrm{Rad}}^{\mathrm{CC}}}{d x d Q^{2}}=\frac{d^{2} \sigma_{\mathrm{Born}}^{\mathrm{CC}}}{d x d Q^{2}}\left[1+\delta_{\mathrm{Rad}}\right]
$$

The full $\mathcal{O}(\alpha)$ radiative corrections may be split into two pieces by writing

$$
1+\delta_{\mathrm{Rad}}=\left(1+\delta_{\gamma}+\delta_{\mathrm{W}}\right) .
$$

A real photon may be radiated from one of the particles involved in the hard scattering or appear as a virtual particle in a one-loop diagram. Such corrections contribute to the 


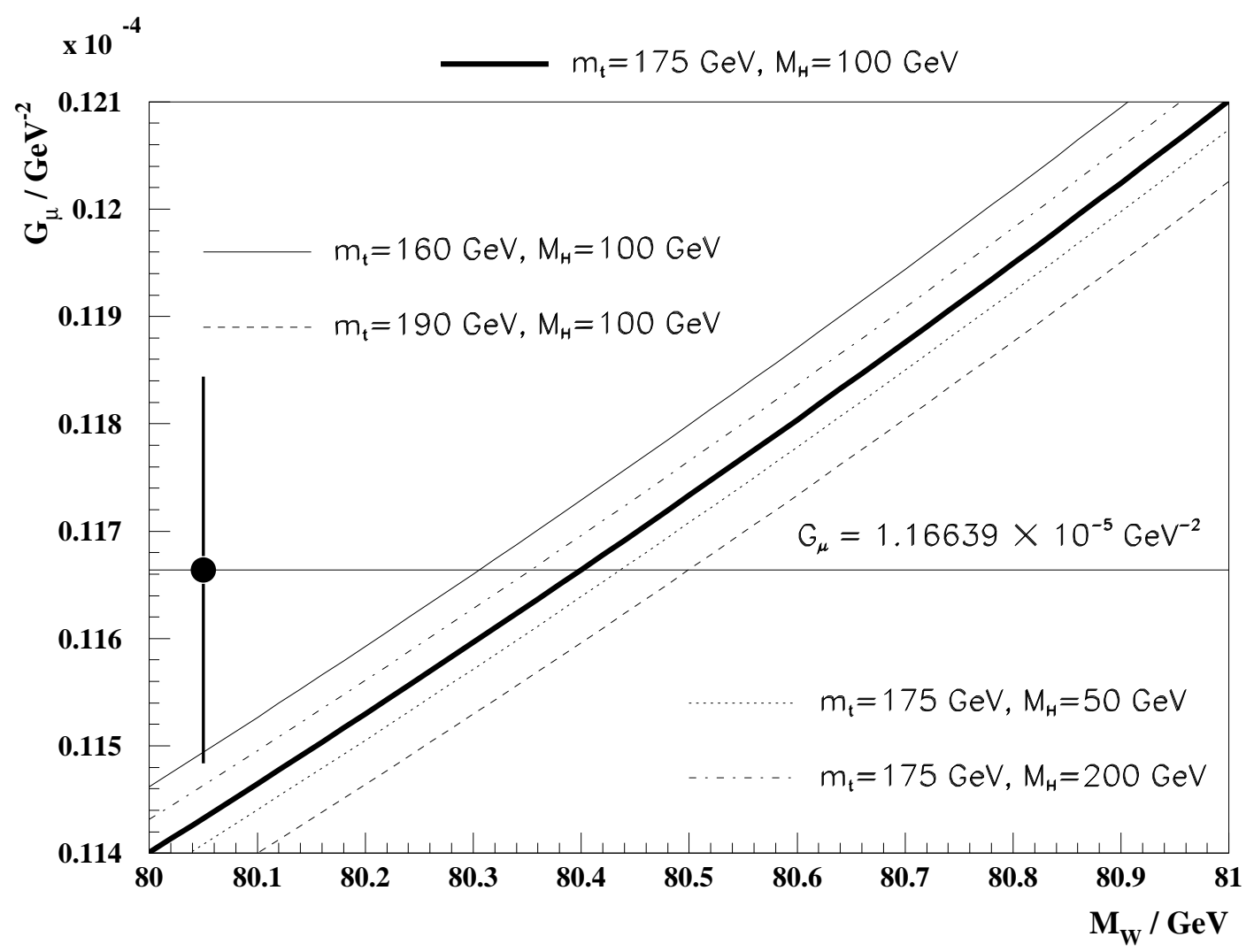

Figure 10. The dependence of $G_{\mu}$ on $M_{W}$ embodied by the $\mathcal{O}(\alpha)$ expression of equation 16. The thick solid line shows the result when $m_{t}$ is set to $175 \mathrm{GeV}$ and $M_{H}$ to $100 \mathrm{GeV}$. The thin solid, dashed, dotted and dash dotted lines show the dependence of $G_{\mu}$ on $M_{W}$ for the values of $m_{t}$ and $M_{H}$ indicated. The point with error bar shows the expected statistical uncertainty on $G_{\mu}$.

term $\delta_{\gamma}$. All other $\mathcal{O}(\alpha)$ contributions are included in the term $\delta_{\mathrm{W}}$. The size of these corrections is shown in Fig 11. The full radiative correction, $\delta_{\text {Rad }}$, was calculated using the program HECTOR [47], while $\delta_{\mathrm{W}}$ was calculated using the program EPRC [45]. It can be seen from Fig 11 that $\delta_{\text {Rad }}$ can be as large as $\pm 20 \%$ while $\delta_{\mathrm{W}}$ is never larger than $\sim 3 \%$.

The radiative corrections to CC DIS depend on $G_{\mu}$; i.e. $\delta=\delta\left(G_{\mu}\right)$, where $\delta$ may be either $\delta_{\mathrm{Rad}}$ or $\delta_{\mathrm{W}}$. Thus for the determination of $G_{\mu}$ (or $M_{W}$ ) from the Born cross section to be valid it is important that $\delta_{\mathrm{Rad}}$ should depend only weakly on $G_{\mu}$. The sensitivity of $\delta_{\mathrm{Rad}}$ and $\delta_{\mathrm{W}}$ to $G_{\mu}$ is shown in Fig 12 where the ratio

$$
R=\frac{1+\delta\left(G_{\mu}=1.226 \times 10^{-} 5 \mathrm{GeV}^{-2}\right)}{1+\delta\left(G_{\mu}=1.166 \times 10^{-} 5 \mathrm{GeV}^{-2}\right)}
$$

is plotted as a function of $x$ for several fixed values of $Q^{2}$. The reference value of $G_{\mu}$ $\left(G_{\mu}=1.166389 \times 10^{-5} \mathrm{GeV}^{-2}\right)$ was taken from reference [51]. Given a statistical error of 
$\sim 1.7 \%$ the value $G_{\mu}=1.226 \times 10^{-5} \mathrm{GeV}^{-2}$ corresponds to raising $G_{\mu}$ by three standard deviations from the reference value. Fig 12 shows that the sensitivity of $\delta_{\mathrm{W}}$ to such a change in $G_{\mu}$ is small $(<1 \%)$. The full radiative correction, $\delta_{\mathrm{Rad}}$, shows a still weaker dependence on $G_{\mu}$ since $\delta_{\text {Rad }}$ is dominated by the photonic corrections contained in $\delta_{\gamma}$.

Given the weak dependence of the radiative corrections on $G_{\mu}$ it is reasonable to estimate the precision with which $G_{\mu}$ (or $M_{W}$ ) may be extracted by making a fit to the Born cross section. In practice, once $G_{\mu}$ has been determined from such a fit, the extraction of the experimental cross section would have to be iterated using the new value of $G_{\mu}$ in order to verify that the results obtained were stable against such modification.

\subsection{Numerical Estimates}

The program HERACLES [53] was used to estimate the statistical error corresponding to a luminosity of $40 \mathrm{pb}^{-1}$ of $e^{+} P$ data (a total of $1020 \mathrm{CC}$ events) distributed among eight bins in $Q^{2}$. The bins were equally spaced in $\log _{10} Q^{2}$ for $Q^{2}$ in the range $400<Q^{2}<40000 \mathrm{GeV}^{2}$. Eq 8 was then used to evaluate $d \sigma_{\mathrm{Born}}^{\mathrm{CC}} / d Q^{2}$. The CTEQ4D PDFs were used through out.

An estimate of the precision with which $G_{\mu}$ may be determined was obtained as follows. The value of $M_{W}$ was fixed at $M_{W}=80.41 \mathrm{GeV}$ and a $\chi^{2}$ fit of the differential cross section, $d \sigma\left(G_{\mu}\right) / d Q^{2}$, performed. This is scenario 1 with $M_{W}$ fixed, as described

in Sec. 田. The fit yielded an error on $G_{\mu}$ of $\pm 0.018 \times 10^{-5} \mathrm{GeV}^{-2}$ which corresponds to $\pm 1.5 \%$. A slightly improved error may be obtained by considering scenario 2 which combines the sensitivity to the propagator and to the normalization into one common parameter, using Eq. 16. The error on $G_{\mu}$ is then $\pm 0.016 \times 10^{-5} \mathrm{GeV}^{-2}( \pm 1.4 \%)$. This last result may be re-expressed to give an estimate of the error on the value of $M_{W}$, using Eq. 19, as suggested in Sec. औ. Such a fit was performed and yielded a statistical error on the parameter $M_{W}$ of $\pm 0.24 \mathrm{GeV}$. These results agree well with similar fits reported in references [43] and [44].

\section{Summary and Outlook}

We now consider what we have learnt and what we can learn given various running conditions: $e^{+} / e^{-}$beams, polarization of the beam, beam energies.

We have seen that HERA data has been used to measure the 'propagator' mass $M_{W}$ and that the precision on such a measurement may be improved by using information on the normalization as well as the shape of the cross-section. Future improvements in luminosity $\left(\sim 1000 \mathrm{pb}^{-1}\right)$ may allow us to achieve an error of $\pm 55 \mathrm{MeV}$ on $M_{W}$ [4]. Sensitivity is greatest with electrons rather than positrons for both $\mathrm{NC}$ and $\mathrm{CC}$ processes and $\mathrm{NC}$ data is most useful with left handed polarization of the lepton beam (which enhances the $\gamma / Z$ terms, see Eqs. 1- 7). Indeed $70 \%$ polarization is worth a factor of 4 in luminosity. 


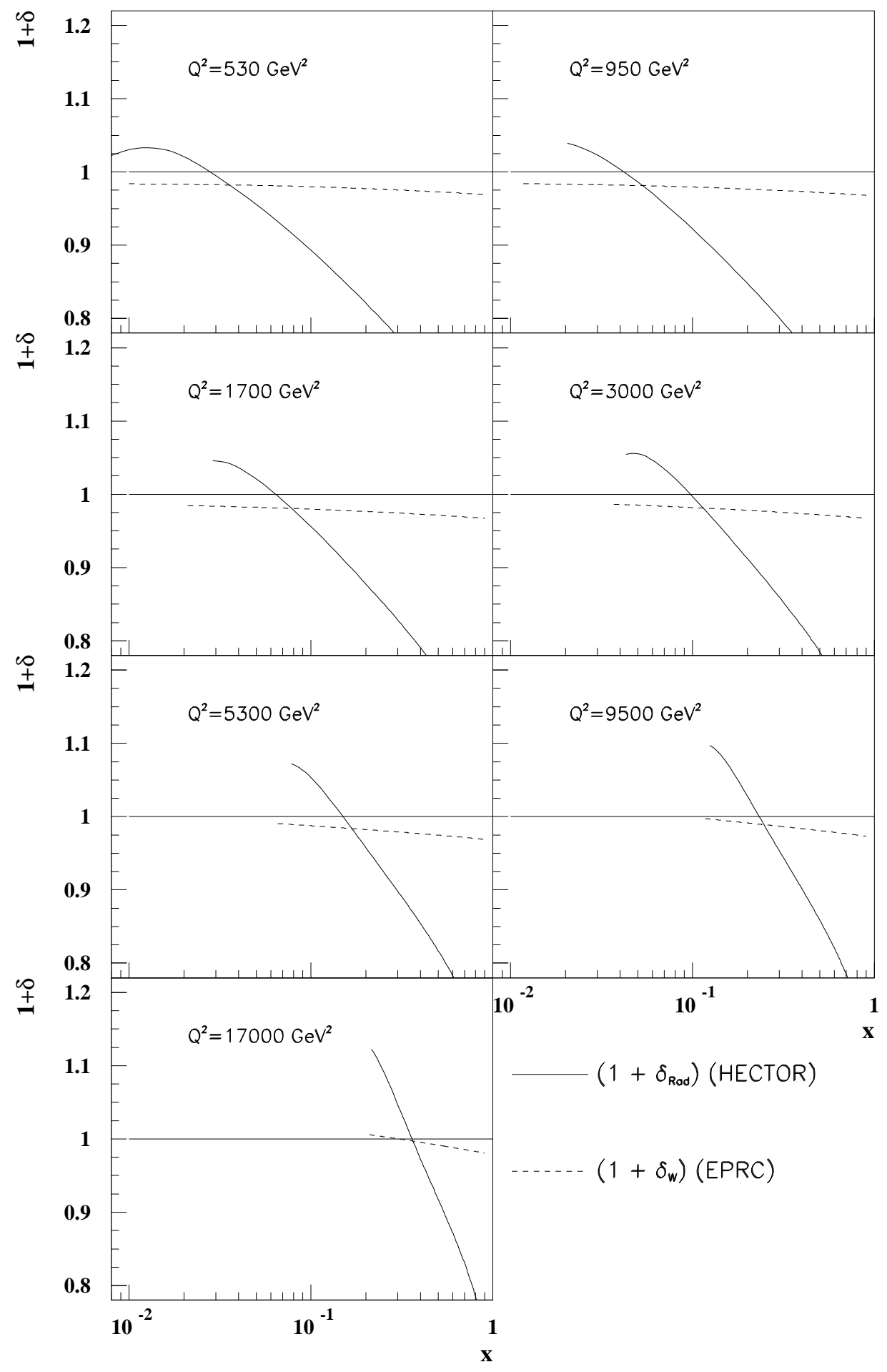

Figure 11. Radiative corrections to charged current deep inelastic scattering. The solid line shows the full radiative correction, $\delta_{\mathrm{Rad}}$, calculated using the program HECTOR [47]. The dashed line shows the electroweak contribution to the radiative corrections, $\delta_{\mathrm{W}}$, calculated using the program EPRC 445]. 


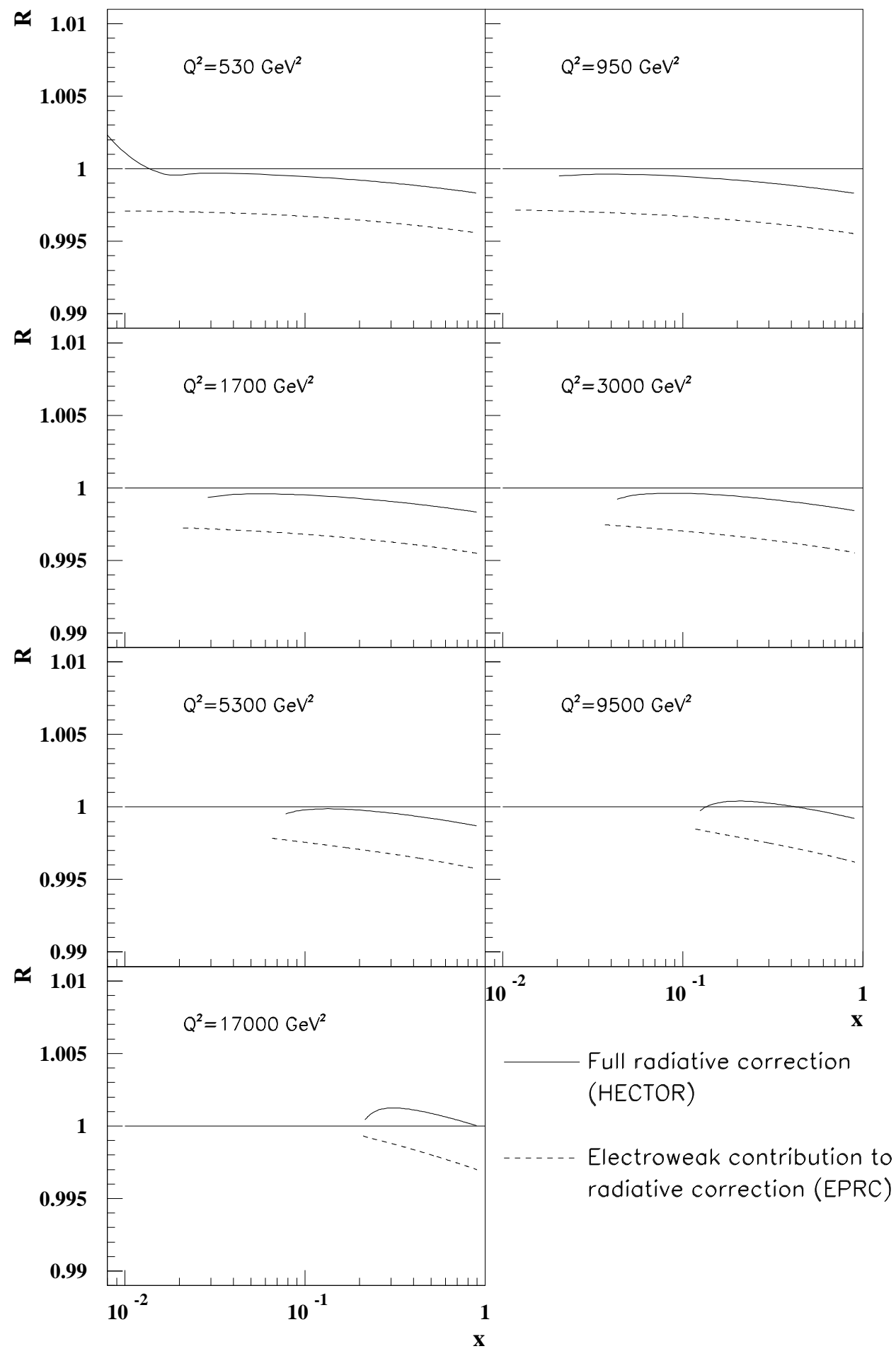

Figure 12. The dependence of the radiative corrections to charged current deep inelastic scattering on the parameter $G_{\mu}$. The ratio $R$, defined in the text, shows the fractional change in the radiative correction as $G_{\mu}$ is varied from $1.116 \times 10^{-} 5 \mathrm{GeV}^{-2}$ to $1.226 \times 10^{-} 5 \mathrm{GeV}^{-2}$. The solid line shows the sensitivity of the full radiative correction (evaluated using HECTOR [47]) to $G_{\mu}$. The dashed line shows the sensitivity of the 
One may also be able to measure the weak neutral current couplings of quarks, $v_{u}$, $a_{u}, v_{d}, a_{d}$ (see Eqs. 1 17), as explored in Ref. [54]. With unpolarized $e^{+}$and $e^{-}$beams ratios of $\mathrm{NC}$ and $\mathrm{CC}$ cross-sections can be used to determine $a_{u}$, whereas polarized beams allow the extraction of $v_{u}$. Measurements of $\mathrm{NC}$ cross-sections alone may be used in a fit to determine all four couplings. Polarization of the beams is essential to achieve reasonable precision. One needs $\simeq 250 \mathrm{pb}^{-1}$ luminosity for each of the four lepton beam charge/polarization combinations to make measurements with a precision of $\sim 10 \%$.

We have seen that HERA CC data can improve our knowledge of the $u$ and $d$ valence distributions (Sec. 3). It seems that knowledge of the $d$ quark is the most crucial since the $u$ quark is already much better known, not being subject to uncertainties due to deuteron binding corrections. To improve our knowledge we will require further positron beam running. Since we would like to explore the valence distributions at the highest possible values of $x$ it may also be worth lowering the proton beam energy to achieve higher $x$ values at the same $Q^{2}\left(x=Q^{2} /(y s)\right)$. This would have two further advantages: it would allow substantial overlap with fixed target data at lower $Q^{2}$ and thus constrain normalizations, and it would allow a model independent measurement of the longitudinal structure function $F_{L}$ [55.

Can we extract other interesting structure functions or PDFs with future high luminosity, high $Q^{2}$, NC and CC data? This has been explored in [56. Polarization is not explicitly useful for this purpose so we will assume $P=0$ in the formulae (though they can be generalized to any value of $P$ ). If we express Eqs. 9 and 10 in terms of reduced cross-sections we obtain

$$
\begin{aligned}
& \tilde{\sigma}_{C C}\left(e^{-} p\right)=\left[x(u+c)+(1-y)^{2} x(\bar{d}+\bar{s})\right] \\
& \tilde{\sigma}_{C C}\left(e^{+} p\right)=\left[x(\bar{u}+\bar{c})+(1-y)^{2} x(d+s)\right] .
\end{aligned}
$$

Then we can define the sum and difference of these reduced cross-sections $\tilde{\sigma}_{ \pm}(C C)=$ $\tilde{\sigma}_{C C}\left(e^{-} p\right) \pm \tilde{\sigma}_{C C}(e+p)$, so that

$$
\begin{aligned}
& \tilde{\sigma}_{+}(C C)=x U+(1-y)^{2} x D \\
& \tilde{\sigma}_{-}(C C)=x u_{v}-(1-y)^{2} x d_{v}
\end{aligned}
$$

where $U$ stands for the sum of the $u$-type quarks and antiquarks $(u, \bar{u}, c, \bar{c}), D$ for the sum of the $d$-type $(d, \bar{d}, s, \bar{s})$ and $u_{v}$ and $d_{v}$ stand for the valence quark densities. Similarly the sum and difference of the $\mathrm{NC} e^{+} p$ and $e^{-} p$ reduced cross-sections give

$$
\begin{aligned}
& \tilde{\sigma}_{+}(N C)=A_{u} x U+A_{d} x D=F_{2} \\
& \tilde{\sigma}_{-}(N C)=B_{u} x u_{v}+B_{d} x d_{v}=x F_{3}
\end{aligned}
$$

where $A_{u}, B_{u}$ are the same for all $u$-type quarks and antiquarks and $A_{d}, B_{d}$ are the same for all $d$-type quarks and antiquarks.

Thus from the CC cross-sections we can mainly gain information on the valence distributions at high $x$, whereas from the NC cross-sections we may measure $x F_{3}$ for 
the first time. We may also consider making combinations of all four cross-sections $\tilde{\sigma}_{+/-}(N C / C C)$ in order to extract $U, D, u_{v}, d_{v}$ for the complete $x$ range. A detailed analysis of this possibility indicates that $200 \mathrm{pb}^{-1}$ per $e^{+} / e^{-}$beam will be necessary to make measurements of $\sim 10 \%$ accuracy [56]. Finally, can HERA shed light on the charm and strange components of the sea? Eqs. 25, 26 indicate how the CC processes may be studied at low $x$ and high and low $y$ to look for evidence of the appropriate sign of $D$ or $K$ hadrons in the final state.

In summary it seems that running conditions with an equal partition between $e^{+} / e-$ and left/right handed polarization will give the most flexibilty for future physics, and that running at a reduced proton beam energy is of interest to physics at high $Q^{2}$ as well as at low $Q^{2}$.

\section{Acknowledgments}

The high $Q^{2}$ working group would like to acknowledge the contribution of all particpants who attended the sessions, with particular thanks to C. Cormack.

\section{References}

[1] C. Adloff et al., Z. Phys. C74, 191 (1997) , J. Breitweg et al., Z. Phys. C74, 207 (1997)

[2] S. Kuhlmann et al., Phys. Lett. B409, 271 (1997)

[3] F. Abe et al., Phys. Rev. Lett. 77, 438 (1996)

[4] C. Pascaud and F. Zomer, preprint LAL 95-05 (1995)

[5] H1 Collab., S. Aid et al., Nucl. Phys. B470 (1996) 3

[6] ZEUS Collab., J. Breitweg et al., DESY 98-121

[7] M. Botje, preprint NIKHEF/97-028, hep-ph/9707289

[8] C. Caso et. al. Eur. Phys. Jour. 3, 1-4 (1998)

[9] J. Blümlein et. al., Proceedings of HERA Workshop 1996, Vol 1 p.23

[10] E. Laenen et al., Nucl. Phys. B392, 162 (1993,) E. Laenen et al., Phys. Lett. B291, 325 (1992), S. Riermersma et al., Phys. Lett. B347, 143 (1995)

[11] H. Lai et. al. Z. Phys. C74, 463 (1997)

[12] A. Martin et. al., RAL-TR-98-029, hep-ph/9803445

[13] M. Buza et. al. Phys. Lett. B411, 211 (1997)

[14] H. Georgi and H.D. Politzer, Phys. Rev. D14, 1829 (1976)

[15] M. Virchaux and A. Milsztajn, Phys. Lett. B274, 221 (1992)

[16] J. Bartels and C. Bontus, these proceedings.

[17] Yu. L. Dokshitzer, Sov. Phys. JETP 46 (1977) 641; V. N. Gribov and L.N. Lipatov, Sov. J. Nucl. Phys. 15 (1972) 438 and 675; G. Altarelli and G. Parisi, Nucl. Phys. B126 (1977) 297

[18] Source code available on request by email to Botje@nikhef.nl, ZEUS Note 97/066

[19] H1 Collaboration, C. Adloff et. al., Z. Phys. C72, 593 (1996)

[20] NMC Collaboration, M. Arneodo et al., Phys. Lett. B364, 107 (1995)

[21] BCDMS Collaboration, A. Benvenuti et al., Phys. Lett. B223, 485 (1989)

[22] L. Frankfurt and M. Strikman, Phys. Rep. 160, 235 (1998)

[23] F. James, CERN Program Library, D506

[24] A. Mehta, these proceedings.

[25] A.Bodek and U.K.Yang, hep-ph/9809480

[26] J. Gomez et al., Phys. Rev. D49, 4348 (1994) 
[27] W. Melnitchouk and A.W. Thomas, Phys. Lett. B377, 11 (1996), W. Melnitchouk and J.C. Peng, Phys. Lett. B400, 220 (1997)

[28] M. Arneodo et al., Nucl. Phys. B487, 3 (1997)

[29] G.R. Farrar and D.R. Jackson, Phys. Rev. Lett. 35, 1416 (1975)

[30] H. Abramowicz et al., Z. Phys. C25, 29 (1984)

[31] F. Abe et al to be submitted to Phys. Rev. Lett., A. Bodek, in proceedings of EW Moriond (1998)

[32] J. Grosse-Knetter et al., in proceedings of ICHEP (1998), T. Doyle, in proceedings of ICHEP (1998)

[33] B. Abbott et al., submitted to Phys. Rev. Lett., J. Huston, in proceedings of ICHEP (1998)

[34] M. Dasgupta and B.R. Webber, Phys. Lett. B382, 273 (1996)

[35] P. Bosted et al., Phys. Rev. D49, 3091 (1994)

[36] E.D. Bloom and F.J. Gilman, Phys. Rev. Lett. 25, 1140 (1970)

[37] H. Spiesberger et al., Proceedings of HERA Workshop 1991, Vol 2 p 798

[38] D. Bardin et al, Z. Phys. C44, 149 (1989)

[39] M. Böhm and H. Spiesberger, Nucl. Phys. B304, 749 (1988)

[40] H. Spiesberger, Nucl. Phys. B349, 109 (1991)

[41] J. Kripfganz and H. Perlt, Z. Phys. C41, 319 (1998)

H. Spiesberger, Phys. Rev. D52, 4936 (1995)

[42] W. Hollik et al., Proceedings of HERA Workshop 1991, Vol 2 p 923

H. Spiesberger, 1995 Precision Tests of the Standard Model Ed. Langacker P (Advanced Series on Directions in High Energy Physics, World Scientific Publishing Co.) p 626

[43] J. Blümlein, M. Klein and T. Riemann, Proceedings of HERA Workshop 1987, Vol 2 p 687

[44] R. Beyer et al., Proceedings of HERA Workshop 1996, Vol 1 p. 140

[45] H. Spiesberger, Proceedings of HERA Workshop 1996, Vol 1 p 227

[46] K. Charchuła, G.A. Schuler and H. Spiesberger, 1994 Comput. Phys. Commun. 81 381; H. Spiesberger, 1998 DJANGOH program manual unpublished

[47] A. Arbuzov et al., Comput. Phys. Commun. 94128 (1996)

[48] Proc. Workshop on HERA Monte Carlo programs 1999 Eds Doyle T, Grindhammer G and Ingelman $\mathrm{G}$, in preparation

[49] H1 Collab., Measurement of Neutral and Charged Current Cross Sections at High $Q^{2}$; Procedings of the XXIX Int. Conf. on HEP, Vancouver, July 1998.

[50] ZEUS Collab., Measurement of High- $Q^{2}$ Charged Current DIS Cross-Sections at HERA Procedings of the XXIX Int. Conf. on HEP, Vancouver, July 1998.

[51] PDG, C. Caso et al., Z. Phys. C3, 1 (1998)

[52] A. Sirlin, Phys. Rev. D22, 971 (1980)

[53] H. Spiesberger, HERACLES version 4.5.2 unpublished program manual http://www.desy.de:8888/ ${ }^{\sim}$ hspiesb/mcp.html

See also: HERACLES 4.4: A. Kwiatowski, H. Spiesberger and H.-J. Möhring,Proceedings of HERA Workshop 1991, Vol 3 p.1294; A. Kwiatowski, H. Spiesberger and H.J. Möhring, Z. Phys. C50 (1991) 165; Comput. Phys. Commun.69 155 (1992)

[54] R. J Cashmore et al., Proceedings of HERA Workshop 1996, Vol. 1 p. 163

[55] A. M. Cooper-Sarkar et al., Proceedings of HERA Workshop 1987, Vol 1 p.235, and Proceedings of HERA Workshop 1991, Vol 1 p.155

[56] G. Ingelman and R.Ruckl, Phys. Lett. B201, 369 (1988) ; Z. Phys. C44, 291 (1989) . 